\title{
Values of special indefinite quadratic forms
}

\author{
by \\ Guido Elsner (Bielefeld)
}

1. Introduction. Let $Q$ denote a $d$-dimensional quadratic form. For $a, b \in \mathbb{R}$ we consider the set $E$ of points in the $d$-dimensional Euclidean space at which $Q$ takes values between $a$ and $b$. In case the quadratic form $Q[x]$ is positive definite, $E$ is an elliptic shell, but in this paper we will investigate indefinite forms, and then $E$ is a hyperbolic shell.

For a (measurable) set $B \subset \mathbb{R}^{d}$ the lattice volume of $B$ is the number of lattice points in $B$ (formally $\operatorname{vol}_{\mathbb{Z}} B:=\#\left(B \cap \mathbb{Z}^{d}\right)$ ), and vol $B$ denotes the Lebesgue measure of $B$. For the hyperbolic shell $E$ we want to approximate its lattice volume by the Lebesgue volume, and estimate a relative lattice point rest of large parts of the hyperbolic shell $E$. Therefore we consider for $r>0$ the $d$-dimensional cube $C_{r}$ with edge length $r$ and intersect the cube $C_{r}$ with the hyperbolic shell $E$. The relative lattice point rest of $E \cap C_{r}$ is now defined by

$$
\Delta:=\left|\frac{\operatorname{vol}_{\mathbb{Z}}\left(E \cap C_{r}\right)-\operatorname{vol}\left(E \cap C_{r}\right)}{\operatorname{vol}\left(E \cap C_{r}\right)}\right| .
$$

We will show, for special indefinite forms $Q$, that $\Delta=\mathcal{O}(1)$ as $r \rightarrow \infty$ (Theorem 2.1) and that even $\Delta=o(1)$ as $r \rightarrow \infty$ provided that $Q$ is irrational (Theorem 2.2). Recall that a quadratic form $Q[x]$ and the corresponding operator $Q$ with non-zero matrix $Q=\left(q_{i j}\right), 1 \leq i, j \leq d$, is called rational if there exists a real number $\lambda \neq 0$ such that the matrix $\lambda Q$ has integer entries only; otherwise it is called irrational.

Similar results for forms $Q$ of signature $(p, q)$ satisfying $\max (p, q) \geq 3$ have been proved by Eskin, Margulis and Mozes in [EMM98]. These are quantitative versions of the well-known Oppenheim problem concerning the distribution of values of $Q[m], m \in \mathbb{Z}^{d}$. In 1929, Oppenheim ([Opp29], [Opp31]) conjectured that if $d \geq 5$ then for an irrational non-degenerate

2000 Mathematics Subject Classification: Primary 11P21.

Key words and phrases: lattice points, ellipsoids, Minkowski's successive minima, rational and irrational indefinite quadratic forms, distribution of values of quadratic forms, Oppenheim conjecture, Davenport-Lewis conjecture. 
quadratic form $Q$ the quantity $m(Q):=\inf \left\{|Q[m]|: m \in \mathbb{Z}^{d}, m \neq 0\right\}$ equals zero. In the rational case this was known by Meyer's theorem (see [Cas78]). Later it was conjectured that $m(Q)=0$ even for $d \geq 3$ and $Q$ irrational (for irrational diagonal forms this was suspected in [DH46] and it is not true in dimensions 3 and 4 without the assumption of irrationality). The different approaches to this and related problems involve various mathematical methods from analytic number theory, ergodic theory, representation theory of Lie groups, reduction theory and the geometry of numbers.

In [Mar89] Margulis established the Oppenheim conjecture in dimensions $d \geq 3$, as stated by Davenport and Heilbronn for $d \geq 5$. In his seminal work he proved that the set of values of $Q$ at lattice points is dense in $\mathbb{R}$. Quantitative versions of this problem were later on developed by Dani and Margulis ([DM93]) and Eskin, Margulis and Mozes ([EMM98]). They consist of quantitative bounds on the ratio between the lattice volume and the Lebesgue volume of the set of points in the cube $C_{r}$ where the quadratic form takes values in a small interval. The quantitative bounds provided in these results yield the asymptotic number of points in these regions as a polynomial in $r$ up to a non-effective error term tending to zero in proportion to the leading term. The estimates thus obtained are implicit, since they do not provide explicit bounds in terms of diophantine approximations of irrational coefficients of the form. For a detailed discussion of results on these problems by Oppenheim, Heilbronn and Davenport and others, see [Mar97]. In [BG99] Bentkus and Götze proved explicit error bounds in the quantitative Oppenheim problem for the elliptic shell as well as for hyperbolic shells for $d \geq 9$ by a common approach. They provide more explicit bounds (in terms of diophantine approximation) for distribution functions of the values of the quadratic form on $C_{r}$, whereas the direct application of the previous methods seems to be restricted to the case of the concentration in compact intervals.

In [Göt04] Götze showed that in the positive definite case for $d \geq 5$ the lattice point rest is of order $\mathcal{O}\left(r^{d-2}\right)$ for arbitrary forms, and of order $o\left(r^{d-2}\right)$ if the form is irrational. These results refine earlier bounds of the same order for dimensions $d \geq 9$ (see also [Göt04] for the history of such estimates and further references).

In the present paper we apply techniques of [Göt04] to special indefinite forms and we obtain explicit bounds in terms of certain Minkowski minima of convex bodies related to these quadratic forms. When adapting these techniques, the main problem is to estimate the difference between the lattice volume and the Lebesgue volume by an integral of generalized theta functions. In order to achieve such an estimate, we develop tools, different from those in [Göt04], which involve adjustable smooth approximations of the indicator functions of the hyperboloid and of the cube $C_{r}$. The bound 
given by an integral of theta functions does not use the special structure of the indefinite forms under consideration. Furthermore, a careful modification of the arguments in [Göt04] even leads to a bound in terms of the Minkowski minima mentioned above, which holds for any indefinite form. The special structure of the forms is only used when we estimate the appearing functions of Minkowski minima by adapting the techniques of [Göt04] to the indefinite case. As in the positive definite case, we show that in the irrational case the maximal gap between successive values of the quadratic form at lattice points converges to 0 as $r$ tends to infinity (Corollary 2.4). Furthermore, we extend the results of Bentkus and Götze ([BG99]) on distribution functions for values of quadratic forms to dimensions from 5 to 8 (Theorem 2.7). In addition, we obtain a result for multivariate diophantine approximations for these special indefinite forms (Theorem 2.6).

This paper is organized as follows: In the second section, we state the two main results about the asymptotics of the relative lattice point rest and derive two important corollaries concerning gaps between values of the quadratic form and concerning multivariate diophantine approximations. Furthermore, we give explicit quantitative bounds for the relative lattice point rest. In the third section, we prove the results of the second section. In the fourth section, we collect auxiliary results (e.g. from geometry of numbers, metric number theory, theory of theta functions), which are used in the proofs of the theorems.

2. Results. Let $\mathbb{R}^{d}, 1 \leq d<\infty$, denote the $d$-dimensional Euclidean space with scalar product $\langle\cdot, \cdot\rangle$ and norm $|\cdot|$ defined by $|x|^{2}=\langle x, x\rangle=$ $x_{1}^{2}+\cdots+x_{d}^{2}$ for $x=\left(x_{1}, \ldots, x_{d}\right) \in \mathbb{R}^{d}$. Let $\mathbb{Z}^{d}$ denote the standard lattice of points with integer coordinates in $\mathbb{R}^{d}$.

Consider the quadratic form

$$
Q[x]:=\langle Q x, x\rangle \quad \text { for } x \in \mathbb{R}^{d},
$$

where $Q: \mathbb{R}^{d} \rightarrow \mathbb{R}^{d}$ denotes a symmetric linear operator in $\operatorname{GL}(d, \mathbb{R})$ with eigenvalues, say, $q_{1}, \ldots, q_{d}$. We write

$$
q_{0}:=\min _{1 \leq j \leq d}\left|q_{j}\right|, \quad q:=\max _{1 \leq j \leq d}\left|q_{j}\right|, \quad \bar{q}:=\max \left\{q_{0}^{-1}, q\right\} .
$$

We always assume that the form is non-degenerate, that is, $q_{0}>0$.

We say that a quadratic form $Q$ is of block-type if $Q=Q^{+}-Q^{-}$, where $Q^{+}$and $Q^{-}$are positive definite quadratic forms, $Q^{+}[x]$ only depends on the first $d_{1}$ coordinates of $\mathbb{R}^{d}$ and $Q^{-}[x]$ on the $d-d_{1}$ remaining ones.

For $a, b \in \mathbb{R}$ with $a \leq b$, and $M \in \mathbb{R}^{d}$, we define

$$
E_{a, b ; M}:=\left\{x \in \mathbb{R}^{d}: a \leq Q[x-M] \leq b\right\} .
$$

Note that if $Q[x]$ is positive definite, then $E_{a, b ; M}$ is an elliptic shell. 
Recall that a quadratic form $Q[x]$ and the corresponding operator $Q$ with non-zero matrix $Q=\left(q_{i j}\right), 1 \leq i, j \leq d$, is called rational if there exists a real number $\lambda \neq 0$ such that the matrix $\lambda Q$ has integer entries only; otherwise it is called irrational.

For $r>0$ we set $C_{r}:=\left\{x \in \mathbb{R}^{d}:|x|_{\infty} \leq r\right\}$, where $|\cdot|_{\infty}$ denotes the maximum norm on $\mathbb{R}^{d}$, and

$$
H_{r, M}:=H_{r, M}^{a, b}:=E_{a, b ; M} \cap C_{r} .
$$

For any (measurable) set $B \subset \mathbb{R}^{d}$ let vol $B$ denote the Lebesgue measure of $B$ and $\operatorname{vol}_{\mathbb{Z}} B$ its lattice volume, that is, the number of lattice points in $B \cap \mathbb{Z}^{d}$. We want to investigate the approximation of the lattice volume of $H_{r, M}$ by the Lebesgue volume. Therefore we estimate the following relative lattice point rest of large parts of hyperbolic shells $H_{r, M}, M \in \mathbb{R}^{d}, r$ large:

$$
\Delta(r, M):=\left|\frac{\operatorname{vol}_{\mathbb{Z}} H_{r, M}-\operatorname{vol} H_{r, M}}{\operatorname{vol} H_{r, M}}\right| .
$$

The two main results of this part of the paper are the following

Theorem 2.1. Let $Q$ be a non-degenerate, d-dimensional, block-type form with $d \geq 5$, and let $M \in \mathbb{R}^{d}$. Then

$$
\Delta(r, M)=\mathcal{O}(1) \quad \text { as } r \rightarrow \infty .
$$

The estimate of Theorem 2.1 refines an explicit bound of order $\mathcal{O}(1)$ obtained for dimensions $d \geq 9$ in [BG97] for arbitrary ellipsoids and in [BG99] for arbitrary hyperbolic shells. Since this bound is optimal in the case of positive definite forms ([Göt04], p. 196), the bound in Theorem 2.1 is also optimal for block-type forms.

In case $Q$ is irrational Theorem 2.1 can be improved.

THEOREM 2.2. Let $Q$ be an irrational non-degenerate d-dimensional block-type form with $d \geq 5$, and let $M \in \mathbb{R}^{d}$. Then

$$
\Delta(r, M)=o(1) \text {. }
$$

For irrational forms and dimension $d \geq 9$ the bound of Theorem 2.2 has already been proved in [BG99]. We should remark again that the bounds of both theorems are explicit and effective.

REMARK 2.3. For $M \in \mathbb{Q}^{d}$ the condition $\Delta(r, M)=o(1)$ implies that $Q$ is irrational.

Using Theorem 2.2 we can easily derive a corollary about gaps between values of block-type forms:

For a positive definite quadratic form, Davenport and Lewis [DL72] conjectured in 1972 that the distance between successive values $v_{n}$ of the quadratic form $Q[x]$ on $\mathbb{Z}^{d}$ converges to zero as $n \rightarrow \infty$, provided that the 
dimension $d$ is at least five and $Q$ is irrational. This conjecture was proved by Götze [Göt04]. Now we can derive an analogous result for irrational block-type forms and dimension $d \geq 5$.

For a vector $M \in \mathbb{R}^{d}$ and a constant $c_{0}>0$ let

$$
V(r):=\left\{Q[x-M]: x \in \mathbb{Z}^{d} \cap C_{r / c_{0}}\right\} \cap\left[-c_{0} r^{2}, c_{0} r^{2}\right]
$$

denote the set of values of $Q[x-M]$, lying in the interval $\left[-c_{0} r^{2}, c_{0} r^{2}\right]$, for lattice points $x \in \mathbb{Z}^{d}$ in a box of edge length $r / c_{0}$.

We define the maximal gap between successive values as

$$
d(r):=\sup _{\substack{u \in V(r) \\(u,+\infty) \cap V(r) \neq \emptyset}} \inf \{v-u: v>u, v \in V(r)\} .
$$

Corollary 2.4. Let $Q$ be a non-degenerate d-dimensional block-type form with $d \geq 5$. Then for $c_{0}$ small enough:

(1) $\lim _{r \rightarrow \infty} d(r)=0$ if $Q$ is irrational.

(2) $\lim _{r \rightarrow \infty} d(r)>0$ if $M \in \mathbb{Q}^{d}$ and $Q$ is rational.

Theorems 2.1 and 2.2 follow from Theorem 2.5 below. Furthermore, in Theorem 2.5 (combined with (3.1) in the proof of Theorems 2.1 and 2.2), estimates of the remainder terms in (2.5) and (2.6) in terms of certain diophantine properties of $Q$ will be given.

In order to describe the explicit bounds we need to introduce some more notations. Let $|(x, y)|_{\infty}$ denote the maximum norm of a vector $(x, y)$ in $\mathbb{R}^{d} \times \mathbb{R}^{d}$. For any $t>0$ and $r \geq 2$ consider the norm $F$ on $\mathbb{R}^{d} \times \mathbb{R}^{d}$ given by

$$
F(x, y):=\left|\left(r(x+t Q y), y r^{-1}\right)\right|_{\infty} .
$$

We introduce the so called Minkowski minima of the convex body $\{F \leq 1\}$ as

$$
M_{1, t}=\inf \left\{F(m, n):(m, n) \in\left(\mathbb{Z}^{d} \times \mathbb{Z}^{d}\right) \backslash 0\right\}
$$

and we define in general $M_{k, t}$ to be the infimum of $\lambda>0$ such that the set of lattice points with norm less than $\lambda$, that is,

$$
\left\{(m, n) \in \mathbb{Z}^{d} \times \mathbb{Z}^{d}: F(m, n)<\lambda\right\},
$$

contains $k$ linearly independent vectors. By definition we have $r M_{k, t} \geq 1$. For $d>4$ and $r \geq 2$ we introduce

$$
\begin{aligned}
& \Gamma_{T, r}:=\inf \left\{r^{d} M_{1, t} \cdots M_{d, t}: T^{-1 /(d-4)} \leq|t| \leq T\right\}, \\
& \rho(r, Q, T):= \bar{q}^{d+1} T^{-1 / 2}+\bar{q}^{3 d / 2} \max \left\{\frac{2}{\pi r}, \frac{\pi}{2 q_{0} q r}, T^{-1 /(d-4)}\right\} \\
&+\bar{q}^{d+2} \Gamma_{T, r}^{-1 / 2+2 / d} \log \left(\bar{q} T^{1 / 2} \Gamma_{T, r}+1\right),
\end{aligned}
$$




$$
\begin{aligned}
& \rho(r, Q):=\inf _{T \geq 1}\left\{(b-a) \bar{q}^{d} q^{-1} T^{-1}+T^{-1 / 2} \bar{q}^{d / 2} q^{-1}\left(2+q^{1 / 2}\right)^{d-2}\right. \\
& \left.\quad+(4 T)^{K}\left(r^{2-d}+q_{0}^{-d / 2} r^{2-d / 2}+\bar{q} r^{2-d / 2}(1+\log r)+\rho(r, Q, T)\right)\right\},
\end{aligned}
$$

where $K$ is a constant chosen according to (3.8) below.

For any fixed $T>1$ and irrational $Q$ it is shown in Lemma 4.23 that

$$
\lim _{r \rightarrow \infty} \Gamma_{T, r}=\infty,
$$

with a rate depending on the diophantine properties of $Q$. This implies that

$$
\lim _{r \rightarrow \infty} \rho(r, Q)=0 .
$$

With these notations we may state a theorem providing quantitative bounds for the difference between the volume and the lattice volume of a hyperbolic shell.

TheOREM 2.5. Let $Q$ be a non-degenerate d-dimensional block-type form with $d \geq 5$, and let $M \in \mathbb{R}^{d}$. Furthermore, let $c(Q, M) \geq 1$ be defined as in Theorem 3.1 below. Then there exist constants $c_{j}>0, j=1,2$, depending on $d$ only and a constant $r_{0}=r(Q, M, a, b)>0$ such that, for any $r \geq r_{0}$,

(1) $\left|\operatorname{vol}_{\mathbb{Z}} H_{r, M}-\operatorname{vol} H_{r, M}\right|$

$$
\leq c_{1} r^{d-2}\left((b-a+1) \bar{q}^{d} q^{-1}+c(Q, M) \bar{q}^{d+1}(\log q+1)+1\right),
$$

(2) $\left|\operatorname{vol}_{\mathbb{Z}} H_{r, M}-\operatorname{vol} H_{r, M}\right| \leq c_{2} r^{d-2}$

$$
\times\left((b-a) \bar{q}^{d+1} q^{-1}\left(|M|+2 q^{-1 / 2} \frac{|a|+|b|}{r}\right) r^{-1}+c(Q, M) \rho(r, Q)\right),
$$

where $\lim _{r \rightarrow \infty} \rho(r, Q)=0$ if $Q$ is irrational.

Note that the summand $\rho(r, Q) r^{d-2}$ in the bound in Theorem 2.5 is at least of order $\mathcal{O}\left(r^{d / 2} \log r\right)$. It may be indeed of this order since $r M_{j, t} \ll_{d} r$ shows that the maximal value of $\Gamma_{T, r}$ is of order $\mathcal{O}\left(r^{d}\right)$ and we may choose $T=\mathcal{O}\left(r^{\bar{\beta}}\right)$ with $\bar{\beta}>0$ sufficiently large.

Note that an error bound of order $r^{d / 2+\varepsilon}$ has been proved by Jarník [Jar28] for diagonal $Q=\operatorname{diag}\left(s_{1}, \ldots, s_{d}\right), s_{j}>0$, for Lebesgue almost all coefficients $s_{j}$.

The proof of Theorem 2.5 is based, roughly speaking, on a "continuous" approximation of $\left|\operatorname{vol}_{\mathbb{Z}} H_{r, M}-\operatorname{vol} H_{r, M}\right|$ by an integral over generalized theta functions. We will derive bounds for parts of this integral, which use the distribution of the first Minkowski minimum $M_{1, t}$. We investigate this distribution using results from metric number theory. As a consequence of this investigation, we also get a result for multivariate diophantine approximation: 
For a vector $x \in \mathbb{R}^{d}$ let $\|x\|:=\inf _{m \in \mathbb{Z}^{d}}|x-m|_{\infty}$ denote the error of integer approximation. For real numbers $t>0$ and $\nu>1$ we introduce

$$
D(t, \nu)=\nu \min \left\{\|t Q n\|: n \in \mathbb{Z}^{d}, 0<|n|_{\infty} \leq \nu\right\},
$$

and let $\lambda$ denote the Lebesgue measure. Then we have

TheOREM 2.6. Let $Q$ be a symmetric, non-degenerate block-type form, normalized so that $q_{0}=1$. Then there exists a constant $c(d)>1$ depending on $d$ only such that for any $r \geq 1,0<\xi-\kappa<1$ and $\nu \geq \tau_{Q}$,

$$
\begin{aligned}
\lambda\left\{t \in[\kappa, \xi]: M_{1, t} \leq \tau\right\} & \leq c(d)\left(q \tau^{2}(\xi-\kappa)+\tau r^{-1}\right), \\
\sup _{t \in[\kappa, \xi]} M_{1, t} & \geq \min \left\{\tau_{Q}, r(\xi-\kappa)\right\}, \\
\sup _{t \in[\kappa, \xi]} D(t, \nu) & \geq \min \left\{\tau_{Q}, \nu(\xi-\kappa) / 2\right\},
\end{aligned}
$$

where

$$
\tau_{Q}:=\left(\frac{c(d)+2}{2 c(d) q}\right)^{1 / 2}
$$

Refining the proofs, we may extend Theorems 2.1 and 2.2 to include the case $a=-\infty$, i.e. the case of distribution functions. This partially extends a result obtained by Bentkus and Götze in [BG99] to dimensions from 5 to 8 .

TheOREM 2.7. Let $Q$ be a non-degenerate, d-dimensional, block-type form with $d \geq 5$, let $M \in \mathbb{R}^{d}$, and set

$$
F_{r, M}(b):=\left\{x \in \mathbb{R}^{d}: Q[x-M] \leq b,|x|_{\infty} \leq r\right\} .
$$

Then

as $r \rightarrow \infty$.

$$
\left|\frac{\operatorname{vol}_{\mathbb{Z}} F_{r, M}(b)-\operatorname{vol} F_{r, M}(b)}{\operatorname{vol} F_{r, M}(b)}\right|= \begin{cases}o(1) & \text { if } Q \text { is irrational, } \\ \mathcal{O}(1) & \text { otherwise, }\end{cases}
$$

3. Proofs. First we deduce Theorems 2.1 and 2.2 from Theorem 2.5:

Proof of Theorems 2.1 and 2.2. By Lemma 4.1, for $M=\left(M_{1}, \ldots, M_{d}\right)$ and $r$ large,

$$
\begin{aligned}
& \operatorname{vol} H_{r, M} \\
& \gg_{d}(b-a) q^{-d / 2}\left(q_{0}^{d / 2}+r^{-1}\left|\left(\left|q_{1}\right|^{1 / 2} M_{1}, \ldots,\left|q_{d}\right|^{1 / 2} M_{d}\right)\right|\right)^{d-2} r^{d-2} \\
& \gg_{d}(b-a) q^{-d / 2} q_{0}^{d(d-2) / 2} r^{d-2} .
\end{aligned}
$$

Dividing the inequalities in Theorem 2.5(1) in the general case (resp. Theorem 2.5(2) in the irrational case) by vol $H_{r, M}$ and using (3.1) completes the proof of Theorem 2.1 (resp. 2.2). 
Proof of Corollary 2.4. If $Q$ is irrational, Theorem 2.5(2) and the estimate (3.1) imply that whenever $c_{0}>0$ is small enough, for any $u, v \in$ $\left[-c_{0} r^{2}, c_{0} r^{2}\right]$ we have

$$
\left|\frac{\operatorname{vol}_{\mathbb{Z}} H_{r, M}^{u, v}}{\operatorname{vol}_{\mathbb{R}} H_{r, M}^{u, v}}-1\right|<\frac{1}{2} \quad \text { as } r \rightarrow \infty .
$$

Hence, $H_{r, M}^{u, v} \cap \mathbb{Z}^{d} \neq \emptyset$ for $r$ sufficiently large. This implies that $\lim _{r \rightarrow \infty} d(r)$ $=0$, proving (1).

If $Q$ is rational, there exists a $\lambda>0$ such that $\lambda Q$ has integer entries. If $M \in \mathbb{Q}^{d}$, there exists a $\mu \in \mathbb{Z}, \mu \neq 0$, such that $\mu M \in \mathbb{Z}^{d}$. Hence, $Q[m-M] \in \lambda^{-1} \mu^{-2} \mathbb{Z}^{d}$ for all $m \in \mathbb{Z}^{d}$. Therefore $d(r) \geq \lambda^{-1} \mu^{-2}>0$ for all $r \geq 1$, which proves (2).

We remark that by using (3.14) and (3.1) one can obtain explicit bounds for $d(r)$ in terms of $r$ and $\rho(r, Q)$, representing diophantine properties of $Q$.

Proof of Remark 2.3. Analyzing the proof of Corollary 2.4(1) we see that $\Delta(r, M)=o(1)$ already implies $\lim _{r \rightarrow \infty} d(r)=0$. If $M \in \mathbb{Q}^{d}$ and $Q$ is rational, then Corollary 2.4(2) yields $\lim _{r \rightarrow \infty} d(r)>0$. Thus, the conditions $M \in \mathbb{Q}^{d}$ and $\Delta(r, M)=o(1)$ force the irrationality of $Q$.

The first step in proving Theorem 2.5 is to analyze smooth approximations of the lattice volume of $H_{r}$.

For $a, b \in \mathbb{R}$ and a smoothing parameter $w>0$ we define $g_{a, b, w}: \mathbb{R} \rightarrow$ $[0,1]$ by

$$
g_{a, b, w}(x):=\frac{1}{w}\left((b+w-x)_{+}-(b-x)_{+}-(a-x)_{+}+(a-w-x)_{+}\right) .
$$

This is a linear continuous approximation of the indicator function $I_{[a, b]}$ of the interval $[a, b]$. By Lemma 4.8 we may rewrite $g_{a, b, w}$ as

$$
\begin{aligned}
g_{a, b, w}(x) & =\frac{1}{2 \pi i} \int_{\beta-i \infty}^{\beta+i \infty}\left(e^{(b+w-x) z}-e^{(b-x) z}-e^{(a-x) z}+e^{(a-w-x) z}\right) \frac{d z}{w z^{2}} \\
& =\frac{1}{2 \pi i} \int_{\beta-i \infty}^{\beta+i \infty} \exp \{-x z\} h_{a, b, w}(z) \frac{d z}{z}
\end{aligned}
$$

where

$$
h_{a, b, w}(z):=\frac{\exp \{w z\}-1}{w z}(\exp \{b z\}-\exp \{(a-w) z\}) .
$$

Using $g_{a, b, w}$ we construct a continuous approximation $V_{w, \varepsilon}^{\mathbb{Z}}(r ; a, b, M)$ of the (monotone) lattice point counting function $r \mapsto \operatorname{vol}_{\mathbb{Z}}\left(H_{r, M}\right)$ depending on two smoothing parameters $w>0$ and $\varepsilon>0$. Setting $Q_{+}:=\left(Q^{T} Q\right)^{1 / 2}$, we 
define

$$
\begin{aligned}
& V_{w, \varepsilon}^{\mathbb{Z}}(r ; a, b, M):=\sum_{x \in \mathbb{Z}^{d}} \exp \left\{-\frac{2}{r^{2}} Q_{+}[x]\right\} g_{a, b, w}(Q[x-M]) \chi_{\varepsilon}\left(\frac{x}{r}\right), \\
& V_{w, \varepsilon}^{\mathbb{R}}(r ; a, b, M):=\int_{\mathbb{R}^{d}} \exp \left\{-\frac{2}{r^{2}} Q_{+}[x]\right\} g_{a, b, w}(Q[x-M]) \chi_{\varepsilon}\left(\frac{x}{r}\right) d x
\end{aligned}
$$

where $\chi_{ \pm \varepsilon}$ is a function with the following properties:

(1) For $u \in \mathbb{R}^{d}$,

$$
\chi_{ \pm \varepsilon}(u)= \begin{cases}\exp \left\{2 Q_{+}[u]\right\} & \text { if }|u|_{\infty} \leq \min \{1,1 \pm \varepsilon\} \\ 0 & \text { if }|u|_{\infty}>\max \{1,1 \pm \varepsilon\}\end{cases}
$$

(2) There exists a constant $c_{1}(Q, M)>0$ such that for

$$
\bar{\chi}_{ \pm \varepsilon}(x):=\chi_{ \pm \varepsilon}(x) \exp \left\{\left\langle x, 2 r^{-1} Q M\right\rangle\right\}
$$

the following estimates hold for an appropriate $K=K(d) \in \mathbb{N}$ :

(a) $\int\left|\hat{\bar{\chi}}_{ \pm \varepsilon}(v)\right| d v \ll_{d} c_{1}(Q, M) \varepsilon^{-K}$,

(b) $\quad \int_{\left\{|v|_{\infty}>d^{-1 / 2} r\right\}}\left|\widehat{\bar{\chi}}_{ \pm \varepsilon}(v)\right| d v \ll_{d} c_{1}(Q, M) \varepsilon^{-K} r^{-1} \quad$ for all $r \geq 1$.

The existence of $\chi_{ \pm \varepsilon}$ follows by standard arguments in Fourier analysis (cf. [Els06, p. 27, Lemma 2.4.5]). Note that the function

$$
\psi_{r, \pm \varepsilon}(x):=\exp \left\{-\frac{2}{r^{2}} Q_{+}[x]\right\} \chi_{ \pm \varepsilon}\left(\frac{x}{r}\right)
$$

approximates the indicator function $I_{\left\{|x|_{\infty} \leq r\right\}}$ and hence the equations $V_{0,0}^{\mathbb{Z}}(r ; a, b, M)=\operatorname{vol}_{\mathbb{Z}}\left(H_{r, M}\right)$ and $V_{0,0}^{\mathbb{R}}(r ; a, b, M)=\operatorname{vol}_{\mathbb{R}}\left(H_{r, M}\right)$ are suggestive.

Proof of Theorem 2.5. Let $0<\varepsilon \leq 1 / 4$. By Lemma 4.4 there exists a constant $c=c(d)>0$ such that

$$
\begin{aligned}
\left|\operatorname{vol}_{\mathbb{Z}} H_{r, M}-\operatorname{vol} H_{r, M}\right| \leq \max \left\{\Delta_{-\varepsilon}, \Delta_{\varepsilon}\right\}+c(b-a) q_{0}^{-d / 2} q^{(d-2) / 2} \\
\times\left(\varepsilon+q_{0}^{-1 / 2} q^{1 / 2}|M| r^{-1}+2 q_{0}^{-1 / 2}(|a|+|b|) r^{-2}\right) r^{d-2},
\end{aligned}
$$

where $\Delta_{ \pm \varepsilon}$ is defined by using (3.9) as follows:

$$
\Delta_{ \pm \varepsilon}:=\left|\int_{\mathbb{R}^{d}} I_{H_{r, M}}(x) \psi_{r, \pm \varepsilon}(x) d x-\sum_{x \in \mathbb{Z}^{d}} I_{H_{r, M}}(x) \psi_{r, \pm \varepsilon}(x)\right|
$$


Hence, by Lemma 4.6,

$$
\begin{gathered}
\Delta_{ \pm \varepsilon} \ll_{d} \max _{ \pm} \sup _{\substack{a^{\prime} \in[a-w, a+w] \\
b^{\prime} \in[b-w, b+w]}}\left|V_{w, \pm \varepsilon}^{\mathbb{R}}\left(r ; a^{\prime}, b^{\prime}, M\right)-V_{w, \pm \varepsilon}^{\mathbb{Z}}\left(r ; a^{\prime}, b^{\prime}, M\right)\right| \\
+8 w q_{0}^{-d / 2} q^{(d-2) / 2}\left(1+\varepsilon+q^{1 / 2} \frac{|M|}{r}\right)^{d-2} r^{d-2}
\end{gathered}
$$

Collecting the estimates (3.10) and (3.12), for $w>0$ and $0<\varepsilon \leq 1 / 4$ we obtain

$$
\begin{aligned}
& \text { (3.13) } \quad r^{2-d}\left|\operatorname{vol}_{\mathbb{Z}} H_{r, M}-\operatorname{vol} H_{r, M}\right| \ll_{d}(b-a) \bar{q}^{d} q^{-1} \varepsilon \\
& +(b-a) \bar{q}^{d+1} q^{-1}\left(\frac{|M|}{r}+2 q^{-1 / 2} \frac{|a|+|b|}{r^{2}}\right)+w \bar{q}^{d} q^{-1}\left(1+\varepsilon+q^{1 / 2} \frac{|M|}{r}\right)^{d-2} \\
& +\max _{ \pm} \sup _{a^{\prime}, b^{\prime}}\left|V_{w, \pm \varepsilon}^{\mathbb{R}}\left(r ; a^{\prime}, b^{\prime}, M\right)-V_{w, \pm \varepsilon}^{\mathbb{Z}}\left(r ; a^{\prime}, b^{\prime}, M\right)\right| r^{2-d} .
\end{aligned}
$$

If we now choose $w=1, \varepsilon=1 / 4$ and $r>r_{0}$ large enough, (3.13) and the result of the crucial Theorem 3.1(1) below yields (note that $d \geq 5$ )

$\left|\operatorname{vol}_{\mathbb{Z}} H_{r, M}-\operatorname{vol} H_{r, M}\right|$

$$
\begin{aligned}
& \ll_{d}(b-a+1) \bar{q}^{d} q^{-1} r^{d-2}+c(Q, M)\left(1+q_{0}^{-d / 2} r^{d / 2}+\bar{q}^{d+1}(\log q+1) r^{d-2}\right) \\
& \ll_{d}\left((b-a+1) \bar{q}^{d} q^{-1}+c(Q, M) \bar{q}^{d+1}(\log q+1)+1\right) r^{d-2}
\end{aligned}
$$

for $r$ large enough. This proves Theorem 2.5(1).

To prove Theorem 2.5(2), for $T \geq 1$ we choose

$$
w:=T^{-1 / 2} \text { and } \quad \varepsilon:=(4 T)^{-1},
$$

and by (3.13) and Theorem 3.1(2) below we deduce that for $r$ sufficiently large,

$$
\begin{aligned}
& r^{2-d}\left|\operatorname{vol}_{\mathbb{Z}} H_{r, M}-\operatorname{vol} H_{r, M}\right| \ll_{d}(b-a) \bar{q}^{d} q^{-1}(4 T)^{-1} \\
& +(b-a) \bar{q}^{d+1} q^{-1}\left(|M|+2 q^{-1 / 2} \frac{|a|+|b|}{r}\right) r^{-1}+T^{-1 / 2} \bar{q}^{d / 2} q^{-1}\left(2+q^{1 / 2}\right)^{d-2} \\
& +c(Q, M)(4 T)^{K}\left(1+q_{0}^{-d / 2} r^{d / 2}+\bar{q}^{d} r^{d / 2}(1+\log r)+r^{d-2} \rho(r, Q, T)\right) r^{2-d} .
\end{aligned}
$$

By taking the infimum over all $T \geq 1$ we get, with (2.13),

$$
\begin{aligned}
& r^{2-d}\left|\operatorname{vol}_{\mathbb{Z}} H_{r, M}-\operatorname{vol} H_{r, M}\right| \\
& \ll_{d}(b-a) \bar{q}^{d+1} q^{-1}\left(|M|+2 q^{-1 / 2} \frac{|a|+|b|}{r}\right) r^{-1}+c(Q, M) \rho(r, Q),
\end{aligned}
$$

which proves Theorem 2.5(2) for an appropriate choice of $r_{0}$. 
The key tool in the previous proofs is the following

THEOREM 3.1. Let $Q$ denote a non-degenerate d-dimensional quadratic form of block-type with $d \geq 5$. Then for all $M \in \mathbb{R}^{d}$ there exist constants $c(Q, M), r_{0}>0$ such that for any $r \geq r_{0}$ and any $T \geq 1$ :

(1) $\left|V_{1, \pm \varepsilon}^{\mathbb{Z}}(r ; a, b, M)-V_{1, \pm \varepsilon}^{\mathbb{R}}(r ; a, b, M)\right|$

$$
\ll_{d} c(Q, M) \varepsilon^{-K}\left(1+2 q_{0}^{-d / 2} r^{d / 2}+\bar{q}^{d+1}(\log q+1) r^{d-2}\right) .
$$

(2) $\left|V_{T^{-1 / 2}, \pm \varepsilon}^{\mathbb{Z}}(r ; a, b, M)-V_{T^{-1 / 2}, \pm \varepsilon}^{\mathbb{R}}(r ; a, b, M)\right| \ll_{d} c(Q, M) \varepsilon^{-K}$

$$
\times\left(1+q_{0}^{-d / 2} r^{d / 2}+\bar{q}^{d} r^{d / 2}(1+\log r)+r^{d-2} \rho(r, Q, T)\right),
$$

where $\rho(r, Q, T)$ is defined in (2.12).

In both inequalities $K=K(d)$ is chosen according to (3.8).

Proof. We want to estimate the difference between these two approximations by integrals of theta functions. By (3.4)-(3.6) we have

$$
\begin{aligned}
& \left|V_{w, \pm \varepsilon}^{\mathbb{Z}}(r ; a, b, M)-V_{w, \pm \varepsilon}^{\mathbb{R}}(r ; a, b, M)\right| \\
& =\mid \sum_{x \in \mathbb{Z}^{d}} \exp \left\{-\frac{2}{r^{2}} Q_{+}[x]\right\} \frac{1}{2 \pi i} \int_{\beta-i \infty}^{\beta+i \infty} \exp \{-z Q[x-M]\} h_{a, b, w}(z) \frac{d z}{z} \chi_{ \pm \varepsilon}\left(\frac{x}{r}\right) \\
& -\int_{\mathbb{R}^{d}} \exp \left\{-\frac{2}{r^{2}} Q_{+}[x]\right\} \frac{1}{2 \pi i} \int_{\beta-i \infty}^{\beta+i \infty} \exp \{-z Q[x-M]\} h_{a, b, w}(z) \frac{d z}{z} \chi_{ \pm \varepsilon}\left(\frac{x}{r}\right) d x \mid .
\end{aligned}
$$

Choosing $\beta=r^{-2}$, decomposing $Q[x-M]=Q[x]+Q[M]-2\langle x, Q M\rangle$ (recall that $Q$ is self-adjoint), and using Fubini's theorem, we get

$$
\begin{aligned}
\left|V_{w, \pm \varepsilon}^{\mathbb{Z}}(r ; a, b, M)-V_{w, \pm \varepsilon}^{\mathbb{R}}(r ; a, b, M)\right| & \mid \int_{r^{-2}-i \infty}^{r^{-2}+i \infty} \exp \{-z Q[M]\} h_{a, b, w}(z) \\
& \times\left\{\sum_{x \in \mathbb{Z}^{d}} \exp \left\{-\frac{2}{r^{2}} Q_{+}[x]-z Q[x]+i\langle x, 2 \operatorname{Im}(z) Q M\rangle\right\} \bar{\chi}_{ \pm \varepsilon}\left(\frac{x}{r}\right)\right. \\
& \left.-\int_{\mathbb{R}^{d}} \exp \left\{-\frac{2}{r^{2}} Q_{+}[x]-z Q[x]+i\langle x, 2 \operatorname{Im}(z) Q M\rangle\right\} \bar{\chi}_{ \pm \varepsilon}\left(\frac{x}{r}\right) d x\right\} \frac{d z}{z} \mid,
\end{aligned}
$$

where $\bar{\chi}_{ \pm \varepsilon}$ is defined as in (3.7).

Since $\bar{\chi}_{ \pm \varepsilon}(x)=(2 \pi)^{-d} \int_{\mathbb{R}^{d}} \widehat{\bar{\chi}}_{ \pm \varepsilon}(v) \exp \{-i\langle x, v\rangle\} d v$ by the Fourier inversion theorem, we obtain 


$$
\begin{aligned}
& \left|V_{w, \pm \varepsilon}^{\mathbb{Z}}(r ; a, b, M)-V_{w, \pm \varepsilon}^{\mathbb{R}}(r ; a, b, M)\right| \\
= & \mid \int_{r^{-2}-i \infty}^{r^{-2}+i \infty} \exp \{-z Q[M]\} h_{a, b, w}(z) \frac{1}{(2 \pi)^{d}} \int_{\mathbb{R}^{d}} \widehat{\bar{\chi}}_{ \pm \varepsilon}(v) \\
& \times\left\{\sum_{x \in \mathbb{Z}^{d}} \exp \left\{-\frac{2}{r^{2}} Q_{+}[x]-z Q[x]+i\left\langle x, 2 \operatorname{Im}(z) Q M-\frac{v}{r}\right\rangle\right\}\right. \\
& \left.-\int_{\mathbb{R}^{d}} \exp \left\{-\frac{2}{r^{2}} Q_{+}[x]-z Q[x]+i\left\langle x, 2 \operatorname{Im}(z) Q M-\frac{v}{r}\right\rangle\right\} d x\right\} d v \frac{d z}{z} \mid .
\end{aligned}
$$

For $v \in \mathbb{C}^{d}$ we introduce the following theta sum and theta integral:

$$
\begin{aligned}
\theta_{v}(z) & :=\exp \{-z Q[M]\} \sum_{x \in \mathbb{Z}^{d}} \exp \left\{-\Theta_{Q, M, r, v}(z, x)\right\}, \\
\theta_{0, v}(z) & :=\exp \{-z Q[M]\} \int_{\mathbb{R}^{d}} \exp \left\{-\Theta_{Q, M, r, v}(z, x)\right\} d x
\end{aligned}
$$

where

$$
\Theta_{Q, M, r, v}(z, x):=\frac{2}{r^{2}} Q_{+}[x]-z Q[x]-i\left\langle x, \frac{v}{r}-2 \operatorname{Im}(z) Q M\right\rangle .
$$

Then we can rewrite (3.15) as follows:

$$
\begin{aligned}
& \left|V_{w, \pm \varepsilon}^{\mathbb{Z}}(r ; a, b, M)-V_{w, \pm \varepsilon}^{\mathbb{R}}(r ; a, b, M)\right| \\
& \quad=\left|\int_{r^{-2}-i \infty}^{r^{-2}+i \infty} h_{a, b, w}(z) \frac{1}{(2 \pi)^{d}} \int_{\mathbb{R}^{d}} \widehat{\bar{\chi}}_{ \pm \varepsilon}(v)\left\{\theta_{v}(z)-\theta_{0, v}(z)\right\} d v \frac{d z}{z}\right| .
\end{aligned}
$$

Consider the segments $J_{0}:=\left[r^{-2}-i / r, r^{-2}+i / r\right]$ and $J_{1}:=\left(r^{-2}+i \mathbb{R}\right) \backslash J_{0}$. Then we may split

$$
\begin{aligned}
& \left|V_{w, \pm \varepsilon}^{\mathbb{Z}}(r ; a, b, M)-V_{w, \pm \varepsilon}^{\mathbb{R}}(r ; a, b, M)\right| \\
& \ll_{d} \mid \int_{J_{0}} h_{a, b, w}(z) \frac{1}{(2 \pi)^{d}} \int_{\mathbb{R}^{d}} \widehat{\bar{\chi}}_{ \pm \varepsilon}(v)\left\{\theta_{v}(z)-\theta_{0, v}(z)\right\} d v \frac{d z}{z} \\
& \quad-\int_{J_{1}} h_{a, b, w}(z) \frac{1}{(2 \pi)^{d}} \int_{\mathbb{R}^{d}} \widehat{\bar{\chi}}_{ \pm \varepsilon}(v) \theta_{0, v}(z) d v \frac{d z}{z} \\
& \quad+\int_{J_{1}} h_{a, b, w}(z) \frac{1}{(2 \pi)^{d}} \int_{\mathbb{R}^{d}} \widehat{\bar{\chi}}_{ \pm \varepsilon}(v) \theta_{v}(z) d v \frac{d z}{z} \mid \\
& =\left|I_{0}-I_{1}+I_{2}\right|, \quad \text { say. }
\end{aligned}
$$


Before estimating these integrals we derive a bound for $h_{a, b, w}\left(r^{-2}+i t\right)$, $t \in \mathbb{R}$. Using

$$
\left|\frac{\exp \left\{w\left(r^{-2}+i t\right)\right\}-1}{w}\right| \leq \min \left\{e\left|r^{-2}+i t\right|, \frac{e+1}{w}\right\}
$$

for $r^{2} \geq \max (w, b)>0, r \geq 1$, we obtain

$$
\left|\frac{h_{a, b, w}\left(r^{-2}+i t\right)}{r^{-2}+i t}\right| \ll\left(e^{b r^{-2}}+e^{a r^{-2}}\right) \frac{1}{w\left|r^{-2}+i t\right|^{2}} \ll \frac{1}{w\left|r^{-2}+i t\right|^{2}},
$$

as well as

$$
\left|\frac{h_{a, b, w}\left(r^{-2}+i t\right)}{r^{-2}+i t}\right| \ll\left(e^{b r^{-2}}+e^{a r^{-2}}\right)\left|r^{-2}+i t\right|^{-1} \ll\left|r^{-2}+i t\right|^{-1} .
$$

Estimation of $I_{0}$. Inequality (3.21) and Lemma 4.12 for $t \in J_{0}$ yield

$$
\begin{aligned}
\Theta_{t}:= & \left|\left(r^{-2}+i t\right)^{-1} h_{a, b, w}\left(r^{-2}+i t\right)\right| \\
& \times\left|\int_{\mathbb{R}^{d}} \widehat{\bar{\chi}}_{ \pm \varepsilon}(v)\left\{\theta_{v}\left(r^{-2}+i t\right)-\theta_{0, v}\left(r^{-2}+i t\right)\right\} d v\right| \\
\ll_{d} & q_{0}^{-d / 2}\left|r^{-2}+i t\right|^{-(d+2) / 2} \exp \left\{-c(Q) \operatorname{Re}\left(\left(r^{-2}+i t\right)^{-1}\right)\right\} \int_{\mathbb{R}^{d}}\left|\widehat{\bar{\chi}}_{ \pm \varepsilon}(v)\right| d v \\
& +2\left|r^{-2}+i t\right|^{-1} \int_{\mathbb{R}^{d}}\left|\widehat{\bar{\chi}}_{ \pm \varepsilon}(v)\right| I_{(r, \infty)}(|v|) d v,
\end{aligned}
$$

where $c(Q)$ is described in Lemma 4.12. Writing

$$
\left|r^{-2}+i t\right|=r^{-2}\left(1+r^{4} t^{2}\right)^{1 / 2} \text { and } \operatorname{Re}\left(\left(r^{-2}+i t\right)^{-1}\right)=r^{2} /\left(1+r^{4} t^{2}\right),
$$

we may introduce the variable $s=\left(1+r^{4} t^{2}\right)^{-1}$ and the function $h(s):=$ $s^{(d+2) / 4} \exp \left\{-c(Q) s r^{2}\right\}$. The maximal value of $h$ on $[0, \infty)$ is attained at $s_{0}=$ $(d+2) /\left(4 c(Q) r^{2}\right)$ and it is bounded by $\left(c(Q) r^{2}\right)^{-(d+2) / 4}$ up to a constant depending on $d$ only.

Using the properties of $\chi_{ \pm \varepsilon}$ (see p. 209) and the fact that $|v| \geq r$ implies $|v|_{\infty} \geq d^{-1 / 2} r$ we now obtain

$$
\begin{aligned}
& \sup _{t \in J_{0}} \Theta_{t} \ll_{d} q_{0}^{-d / 2} r^{d+2} \sup _{s \geq 0} h(s) \int_{\mathbb{R}^{d}}\left|\widehat{\bar{\chi}}_{ \pm \varepsilon}(v)\right| d v+2 r^{2} \int_{\mathbb{R}^{d}}\left|\widehat{\bar{\chi}}_{ \pm \varepsilon}(v)\right| I_{(r, \infty)}(|v|) d v \\
& \ll_{d} q_{0}^{-d / 2} r^{d+2}\left(c(Q) r^{2}\right)^{-(d+2) / 4} \int_{\mathbb{R}^{d}}\left|\widehat{\bar{\chi}}_{ \pm \varepsilon}(v)\right| d v \\
& \quad+2 r^{2} \int_{\mathbb{R}^{d}}\left|\widehat{\bar{\chi}}_{ \pm \varepsilon}(v)\right| I_{\left(d^{-1 / 2} r, \infty\right)}\left(|v|_{\infty}\right) d v \\
& \ll_{d} q_{0}^{-d / 2} r^{d+2}\left(c(Q) r^{2}\right)^{-(d+2) / 4} c_{1}(Q, M) \varepsilon^{-K}+c_{1}(Q, M) \varepsilon^{-K} r .
\end{aligned}
$$


Integrating this bound over $J_{0}$, for a suitably chosen constant $c_{2}(Q, M)>0$ we get

$$
\left|I_{0}\right| \leq \int_{-1 / r}^{1 / r} \Theta_{t} d t \ll_{d} c_{2}(Q, M) \varepsilon^{-K} q_{0}^{-d / 2} r^{d / 2}+c_{1}(Q, M) \varepsilon^{-K} .
$$

Estimation of $I_{1}$. Using Lemma 4.11, (4.13) and (4.15), we have

$$
\left|\theta_{0, v}(z)\right| \ll_{d} q_{0}^{-d / 2}|z|^{-d / 2} .
$$

Therefore, by the properties of $\chi_{ \pm \varepsilon}$ (p. 209) and (3.21) for $r^{2} \geq \max \{w, b, 1\}$ we get

$$
\begin{aligned}
\left|I_{1}\right| & \ll_{d} q_{0}^{-d / 2} c_{1}(Q, M) \varepsilon^{-K} \int_{J_{1}}\left|\left(r^{-2}+i t\right)^{-(1+d / 2)}\right| d t \\
& \ll_{d} q_{0}^{-d / 2} c_{1}(Q, M) \varepsilon^{-K} \int_{1 / r}^{\infty} t^{-(1+d / 2)} d t \\
& \ll_{d} q_{0}^{-d / 2} c_{1}(Q, M) \varepsilon^{-K} r^{d / 2},
\end{aligned}
$$

using the symmetry in $t$ around 0 .

Estimation of $I_{2}$. From (3.20) and (3.21) we obtain $\left|h_{a, b, w}\left(r^{-2}+i t\right)\right| \ll_{d}$ $\min \left\{1,\left(\left|r^{-2}+i t\right| w\right)^{-1}\right\}$, which implies

$$
\begin{aligned}
& \ll_{d} \int_{\mathbb{R}^{d}|t|>1 / r} \int_{I_{2} \mid}\left|\theta_{v}\left(\frac{1}{r^{2}}+i t\right)\right| \min \left\{1, \frac{1}{w\left|r^{-2}+i t\right|}\right\} \frac{d t}{\left|r^{-2}+i t\right|}\left|\widehat{\bar{\chi}}_{ \pm \varepsilon}(v)\right| d v \\
& \ll_{d} \int_{\mathbb{R}^{d}|u|>2 / \pi r}\left|\theta_{v}\left(r^{-2}+i \pi u / 2\right)\right| g(u) d u\left|\widehat{\bar{\chi}}_{ \pm \varepsilon}(v)\right| d v
\end{aligned}
$$

where

$$
g(u)=\min \left\{1,(w|u|)^{-1}\right\}|u|^{-1} .
$$

Using Lemma 4.15 and the properties of $\chi_{ \pm \varepsilon}$ (see p. 209), we have

$$
\begin{aligned}
\left|I_{2}\right| & \ll_{d} \bar{q}^{d} r^{d / 2} \int_{\mathbb{R}^{d}} \int_{|u|>2 / \pi r}\left(M_{1, t} \cdots M_{d, t}\right)^{-1 / 2} g(u) d u\left|\widehat{\bar{\chi}}_{ \pm \varepsilon}(v)\right| d v \\
& \ll_{d} \bar{q}^{d} r^{d / 2} c_{1}(Q, M) \varepsilon^{-K} \int_{|u|>2 / \pi r}\left(M_{1, t} \cdots M_{d, t}\right)^{-1 / 2} g(u) d u
\end{aligned}
$$

where $M_{j, t}$ denote Minkowski's successive minima for the norm on $\mathbb{R}^{2 d}$ related to $Q$, defined by (4.27) and (4.30), and $c_{1}(Q, M)>0$ is a constant 
chosen according to (3.7). Define

$$
G(\kappa, \xi):=\int_{\kappa}^{\xi} g(t) d t \quad \text { for } 0<\kappa<\xi \leq \infty .
$$

For $\kappa \geq \xi>0$ we define $G(\kappa, \xi)=0$. Note that

$$
G(\kappa, \xi)= \begin{cases}\log (\xi / \kappa) & \text { for } \kappa \leq \xi \leq w^{-1} \\ -\log (w \kappa)+1-(w \xi)^{-1} & \text { for } \kappa \leq w^{-1} \leq \xi, \\ (w \kappa)^{-1}-(w \xi)^{-1} & \text { for } w^{-1} \leq \kappa \leq \xi\end{cases}
$$

The equality (3.29) and the definition of $G$ imply

$$
G(\kappa, \xi) \leq \min \left\{|\log (w \kappa)|+1,|\log (\xi / \kappa)|,(w \kappa)^{-1}\right\} \quad \text { for } \kappa, \xi>0 .
$$

If we write $M(t)=M_{1, t} \cdots M_{d, t}$, the upper bound for $\left|I_{2}\right|$ in (3.27) in terms of Minkowski's successive minima yields

$$
\begin{aligned}
& \left|I_{2}\right| \ll_{d} \bar{q}^{d} r^{d / 2} c_{1}(Q, M) \varepsilon^{-K} \int_{|t|>2 / \pi r} \frac{g(t)}{M(t)^{1 / 2}} d t \\
& =2 \bar{q}^{d} r^{d / 2} c_{1}(Q, M) \varepsilon^{-K} I_{3},
\end{aligned}
$$

where

$$
I_{3}=\int_{2 / \pi r}^{\infty} \frac{g(t)}{M(t)^{1 / 2}} d t
$$

The last equality in (3.31) follows from the fact that the functions $g(\cdot)$ and $M(\cdot)$ are even (see (4.33)).

After these preparations, we may now complete the proof of Theorem 3.1:

Proof of Theorem 3.1(1). Let

$$
\gamma(\kappa, \xi)=r^{d} \inf _{\kappa \leq t \leq \xi} M(t) \quad \text { for } \kappa, \xi \in \mathbb{R} .
$$

Applying Lemma 4.22 for the interval with endpoints $\kappa=2 / \pi r$ and $\xi=\infty$, we get

$$
\begin{aligned}
& I_{3} \ll_{d} q_{0}^{-1} r^{d / 2-2} \int_{\gamma_{0}}^{D_{0}} v^{-1 / 2+1 / d}\left(q v^{1 / d} G\left(\kappa_{0}\left(v^{1 / d}\right), \infty\right)+g\left(\kappa_{0}\left(v^{1 / d}\right)\right)\right) \frac{d v}{v} \\
& \quad+G\left(\frac{2}{\pi r}, \infty\right)
\end{aligned}
$$


with

$$
\begin{aligned}
\gamma_{0} & =\gamma\left(\frac{2}{\pi r}, \infty\right), \quad D_{0}=\max \left\{\left(\frac{r}{2 d}\right)^{d}, \gamma_{0}\right\}, \\
\kappa_{0}(v) & =\max \left\{\frac{2}{\pi r}, \frac{1}{2 q v d^{1 / 2}}\right\} .
\end{aligned}
$$

Note that $\gamma_{0} \geq 1$ by (4.29). In the following we choose $w=1$. Using $(3.26)$, (3.30), (3.31), (3.34), (3.35) and hence $g\left(\kappa_{0}\left(v^{1 / d}\right)\right) \ll_{d} q v^{1 / d}$, we obtain for $d>4$ and $r \geq \max \{2 / \pi q, 2 / \pi\}$,

$$
\begin{aligned}
& \left|I_{2}\right| \ll_{d} c_{1}(Q, M) \varepsilon^{-K} \bar{q}^{d+1} r^{d / 2} r^{d / 2-2} \int_{1}^{D_{0}} v^{-1 / 2+2 / d}\left(\log \left(q v^{1 / d}\right)+2\right) \frac{d v}{v} \\
& +c_{1}(Q, M) \varepsilon^{-K} \bar{q}^{d} r^{d / 2}(\log r+1) \\
& \ll_{d} c_{1}(Q, M) \varepsilon^{-K} \bar{q}^{d+1}(\log q+1) r^{d-2} .
\end{aligned}
$$

For $r \geq r_{0}:=\max \left\{2 / \pi q, 2 / \pi, r_{0}(Q, M)\right\}$, where $r_{0}(Q, M)$ is a constant chosen as in Lemmas 4.12 and 4.13, this bound for $I_{2}$ implies, in view of (3.18), (3.22) and (3.24), that

$$
\begin{aligned}
& \left|V_{1, \pm \varepsilon}^{\mathbb{Z}}(r ; a, b, M)-V_{1, \pm \varepsilon}^{\mathbb{R}}(r ; a, b, M)\right| \ll_{d} c_{2}(Q, M) \varepsilon^{-K} q_{0}^{-d / 2} r^{d / 2} \\
& \quad+c_{1}(Q, M) \varepsilon^{-K}\left(1+q_{0}^{-d / 2} r^{d / 2}+\bar{q}^{d+1}(\log q+1) r^{d-2}\right)
\end{aligned}
$$

where the constants $c_{1}(Q, M)$ and $c_{2}(Q, M)$ are chosen according to Lemma 3.7 and (3.22). Setting $c(Q, M):=\max \left\{c_{1}(Q, M), c_{2}(Q, M)\right\}$ proves Theorem 3.1(1).

Proof of Theorem 3.1(2). In order to use non-trivial bounds for $\gamma(\kappa, \xi)$ in the irrational case we introduce further auxiliary parameters $\eta, T$ such that $2 / \pi r \leq \eta \leq T$ with $T \geq 1$ which will be determined and optimized later. Thus we may split the integral $I_{3}$ in (3.32) which bounds $\left|I_{2}\right|$ in (3.31) into the parts

$$
\begin{aligned}
I_{3} & =\int_{2 / \pi r}^{\eta} \frac{g(t)}{M(t)^{1 / 2}} d t+\int_{\eta}^{T} \frac{g(t)}{M(t)^{1 / 2}} d t+\int_{T}^{\infty} \frac{g(t)}{M(t)^{1 / 2}} d t \\
& =I_{4}+I_{5}+I_{6}, \quad \text { say. }
\end{aligned}
$$

We define, similarly to (3.35),

$$
\begin{aligned}
& \gamma_{1}= \gamma(2 / \pi r, \eta), \quad \gamma_{2}=\gamma(\eta, T), \quad \gamma_{3}=\gamma(T, \infty), \\
& D_{j}=\max \left\{(2 d)^{-d_{r}}, \gamma_{j}\right\}, \quad j=1,2,3 \\
& \kappa_{1}(v)=\max \{2 / \pi r, f(v)\}, \quad \kappa_{2}(v)=\max \{\eta, f(v)\} \\
& \kappa_{3}(v)=\max \{T, f(v)\},
\end{aligned}
$$


where $f(v)=\left(2 q v d^{1 / 2}\right)^{-1}, v>0$. By (4.29) we have again

$$
\gamma_{j} \geq 1, \quad j=1,2,3 .
$$

Using (3.26) and (3.40), we see that

$$
g\left(\kappa_{j}(v)\right) \leq 2 q v d^{1 / 2}, \quad j=1,2,3 .
$$

First, we apply Lemma 4.22 as above to the interval with endpoints $\kappa=2 / \pi r$ and $\xi=\eta$. Corollary 4.17 implies that if $\eta \geq \pi / 2 q_{0} q r$ the quantity $\gamma_{1}$ (defined by (3.33) and (3.38)) satisfies

$$
\gamma_{1} \geq \delta:=(d q \eta)^{-d}
$$

since $d \geq 5$ and

$$
\inf _{t \in[2 / \pi r, \eta]}\left\{\frac{q_{0}|t| r}{2}, \frac{1}{q|t| r}\right\}=\frac{1}{q \eta r} \quad \text { whenever } \quad \eta \geq \frac{\pi}{2 q_{0} q r} .
$$

In view of (3.29), (3.30), (3.42) and (3.43) Lemma 4.22 yields the estimate

$$
\begin{aligned}
I_{4} & \ll_{d} q_{0}^{-1} r^{d / 2-2} \int_{\gamma_{1}}^{D_{1}} v^{-1 / 2+1 / d}\left(v^{1 / d} q G\left(\kappa_{1}\left(v^{1 / d}\right), \eta\right)\right. \\
& \left.+g\left(\kappa_{1}\left(v^{1 / d}\right)\right)\right) \frac{d v}{v}+G(2 / \pi r, \eta) \\
& \ll_{d} q_{0}^{-1} q r^{d / 2-2} \int_{\delta}^{D_{1}} v^{-1 / 2+2 / d}\left(\left|\log \left(q v^{1 / d} \eta\right)\right|+1\right) \frac{d v}{v}+G(2 / \pi r, \eta) \\
& \ll_{d} q_{0}^{-1} q^{d / 2-1} r^{d / 2-2} \eta^{d / 2-2}+G(2 / \pi r, \eta),
\end{aligned}
$$

provided that $d>4$, using the change of variables $v=\delta u$ in the last inequality.

In order to estimate $I_{5}$ we choose $\kappa=\eta$ and $\xi=T$. By Lemma 4.22 we obtain as above

$$
\begin{aligned}
I_{5} & \ll_{d} q_{0}^{-1} r^{d / 2-2} \int_{\gamma_{2}}^{D_{2}} v^{-1 / 2+1 / d}\left(v^{1 / d} q G\left(\kappa_{2}\left(v^{1 / d}\right), T\right)\right. \\
& +g\left(\kappa_{2}\left(v^{1 / d}\right)\right) \frac{d v}{v}+G(\eta, T) \\
& \ll_{d} q_{0}^{-1} q r^{d / 2-2} \int_{\gamma_{2}}^{D_{2}} v^{-1 / 2+2 / d}\left(\left|\log \left(q v^{1 / d} / w\right)\right|+1\right) \frac{d v}{v}+G(\eta, T) \\
& \ll_{d} q_{0}^{-1} q r^{d / 2-2} \gamma_{2}^{-1 / 2+2 / d}\left(\left|\log \left(q \gamma_{2}\right)\right|+|\log w|+1\right)+G(\eta, T) .
\end{aligned}
$$

Finally, for the term $I_{6}$ choose $\kappa=T$ and $\xi=\infty$ and use (3.41) for $j=3$. Recall that we choose $T \geq 1$. Thus, as above, using Lemma 4.22 and the fact that $G\left(\kappa_{3}\left(v^{1 / d}\right), \infty\right) \leq G(T, \infty) \leq T^{-1} w^{-1}$ and $g\left(\kappa_{3}\left(v^{1 / d}\right)\right) \leq T^{-2} w^{-1}$, 
we obtain (see (3.26), (3.30) and (3.40))

$$
\begin{aligned}
I_{6} & \ll_{d} q_{0}^{-1} r^{d / 2-2} \int_{1}^{D_{3}} v^{-1 / 2+1 / d}\left(v^{1 / d} q G\left(\kappa_{3}\left(v^{1 / d}\right), \infty\right)\right. \\
& \left.+g\left(\kappa_{3}\left(v^{1 / d}\right)\right)\right) \frac{d v}{v}+G(T, \infty) \\
& \ll_{d} q_{0}^{-1} q r^{d / 2-2} T^{-1} w^{-1}+G(T, \infty) .
\end{aligned}
$$

Collecting (3.44)-(3.46), by combining the terms $G(\kappa, \xi)$ and using (3.37) and the estimates $(3.41)$ we get

$$
\begin{aligned}
I_{3} \ll_{d} q_{0}^{-1} r^{d / 2-2}\left\{q^{d / 2-1} \eta^{d / 2-2}\right. & +q \gamma_{2}^{-1 / 2+2 / d}\left(\log \left(q \gamma_{2}\right)\right. \\
& \left.+|\log w|+1)+\frac{q}{T w}\right\}+G(2 / \pi r, \infty) .
\end{aligned}
$$

In view of (3.31) this bound for $I_{3}$ yields

$$
\begin{array}{rl}
\left|I_{2}\right| \ll_{d} & c_{1}(Q, M) \varepsilon^{-K} q^{d} r^{d / 2}(1+\log r)+c_{1}(Q, M) \varepsilon^{-K} q_{0}^{-1} \bar{q}^{d} r^{d-2} \\
& \times\left\{(T w)^{-1}+q^{d / 2-1} \eta^{d / 2-2}+q \gamma_{2}^{-1 / 2+2 / d}\left(\log \left(q \gamma_{2}\right)+|\log w|+1\right)\right\} \\
\ll_{d} & c(Q, M) \varepsilon^{-K} \bar{q}^{d} r^{d / 2}(1+\log r)+c(Q, M) \varepsilon^{-K} r^{d-2} \\
& \times\left\{\frac{\bar{q}^{d+1}}{T w}+\bar{q}^{3 d / 2} \eta^{d / 2-2}+\bar{q}^{d+2} \gamma_{2}^{-1 / 2+2 / d}\left(\log \left(\bar{q} \gamma_{2}\right)+|\log w|+1\right)\right\},
\end{array}
$$

where $c(Q, M):=\max \left\{c_{1}(Q, M), c_{2}(Q, M)\right\}$. By Lemma 4.23 for $\eta, T$ fixed, we have $\gamma_{2} \rightarrow \infty$ as $r \rightarrow \infty$ and we may now choose the auxiliary parameters $\eta, w$ and $T$ to minimize the right hand side of (3.48) as follows. Let

(3.49) $\quad T \geq 1, \quad w=T^{-1 / 2}, \quad \eta=\max \left\{\frac{2}{\pi r}, \frac{\pi}{2 q_{0} q r}, T^{-1 /(d-4)}\right\}$,

provided that $d \geq 5$.

For $r \geq r_{0}:=\max \left\{2 / \pi, \pi / 2 q_{0} q, r_{0}(Q, M)\right\}$, where $r_{0}(Q, M)$ is a constant chosen as in Lemmas 4.12 and 4.13, in view of (3.18), (3.22), (3.24), (3.38), (3.41), (3.48) and (3.49) we obtain the following bound:

$$
\begin{aligned}
& \left|V_{T^{-1 / 2}, \varepsilon}^{\mathbb{Z}}(r ; a, b, M)-V_{T^{-1 / 2}, \varepsilon}^{\mathbb{R}}(r ; a, b, M)\right| \\
& \quad \ll_{d} c(Q, M) \varepsilon^{-K}\left(1+q_{0}^{-d / 2} r^{d / 2}+\bar{q}^{d} r^{d / 2}(1+\log r)+r^{d-2} \rho(r, Q, T)\right),
\end{aligned}
$$

where $\rho(r, Q, T)$ is defined as in (2.12). This completes the proof of Theorem $3.1(2)$.

Proof of Theorem 2.6. The estimate (2.17) immediately follows from Corollary 4.21. This inequality ensures that there exists a $t \in[\kappa, \xi]$ such 
that $M_{1, t}>\tau$ whenever $c(d)\left(q \tau^{2}(\xi-\kappa)+\tau r^{-1}\right)<\xi-\kappa$. This condition is equivalent to

$$
\tau<\left(\frac{1}{c(d)}-q \tau^{2}\right)(\xi-\kappa) r .
$$

Since $\tau \leq \tau_{Q}$, where $\tau_{Q}:=((c(d)+2) / 2 c(d) q)^{1 / 2}$, implies $1 / c(d)-q \tau^{2} \geq 1 / 2$, we may conclude that the condition (3.50) (and hence $M_{1, t}>\tau$ ) follows from the inequality $\tau \leq \min \left\{\tau_{Q}, r(\xi-\kappa) / 2\right\}$, which proves (2.18).

By definition of $M_{1, t}$ the inequality $M_{1, t}>\bar{\tau}:=\min \left\{\tau_{Q}, r(\xi-\kappa) / 2\right\}$ implies that if $0<|n|_{\infty}<\bar{\tau} r$ then $\bar{\tau} r\|t Q n\|>\bar{\tau}^{2}$. For $\nu>\tau_{Q}$ there exists an $r \geq 1$ such that $\nu=\bar{\tau} r$. Therefore, by $(2.16)$ we get $D(t, v) \geq \bar{\tau}^{2}$. Furthermore, we have $\bar{\tau}^{2}=\min \left\{\tau_{Q}^{2}, \nu(\xi-\kappa) / 2\right\}$, since either $r(\xi-\kappa) / 2>\tau_{Q}$ and $\bar{\tau}=\tau_{Q}$, or $\bar{\tau}=r(\xi-\kappa) / 2$. This proves $(2.19)$.

Proof of Theorem 2.7. Since the cube $C_{r}$ is compact the quantity

$$
a_{r}:=\min \left\{Q[x-M]: x \in C_{r}\right\}
$$

is a well-defined real number and we obviously get

$$
F_{r, M}(b)=H_{r, M}^{a_{r}, b},
$$

where $H_{r, M}^{a_{r}, b}$ is defined as in (2.3).

A careful analysis of the proof shows that Theorem 3.1 also holds for $a=a_{r}, r \geq r_{0}$. This, together with Lemma 4.7 for $\varepsilon=(4 T)^{-1}, T \geq 1$, implies that there exist constants $c_{j}>0, j=1,2,3$, depending on $Q$ and $d$ only, and a constant $r_{0}=r_{0}(Q, M, b)>0$ such that, for any $r \geq r_{0}$ (cf. proof of Theorem 2.5):

(1) $\left|\operatorname{vol}_{\mathbb{Z}} F_{r, M}(b)-\operatorname{vol} F_{r, M}(b)\right| \leq r^{d-2}\left(c_{1}\left(b-a_{r}+1\right)+c_{2}\right)$.

$(2)\left|\operatorname{vol}_{\mathbb{Z}} F_{r, M}(b)-\operatorname{vol} F_{r, M}(b)\right| \leq r^{d-2}\left(c_{3}\left(b-a_{r}\right) \tilde{\rho}(r, Q)\right)$,

where

$$
\begin{aligned}
\tilde{\rho}(r, Q):= & \inf _{T \geq 1}\left\{T^{-1}+\frac{T^{-1 / 2}\left(1+T^{-1}\right)^{d-2}}{b-a_{r}}\right. \\
& \left.+\frac{(4 T)^{K}}{b-a_{r}}\left(r^{2-d}+q_{0}^{-d / 2} r^{2-d / 2}+\bar{q} r^{2-d / 2}(1+\log r)+\rho(r, Q, T)\right)\right\},
\end{aligned}
$$

and $\lim _{r \rightarrow \infty} \tilde{\rho}(r, Q)=0$ if $Q$ is irrational.

In the definition of $\tilde{\rho}(r, Q)$ the constant $K$ is chosen according to (3.8).

Dividing these inequalities by the inequality in Lemma 4.3(2) for $\xi=1$ completes the proof of Theorem 2.7.

4. Lemmas. Let $I=[a, b], a, b \in \mathbb{R}$, and $I_{0}$ denote finite intervals. For $M \in \mathbb{R}^{d}$ we consider

$$
H(r):=H\left(r, I_{0}, I, M\right):=\left\{x \in \mathbb{R}^{d}: r^{-1}|x|_{\infty} \in I_{0}, Q[x-M] \in I\right\} .
$$


The diagonal matrix $D(Q)$ is defined by

$$
(D(Q))_{i, i}:=\sqrt{\left|q_{i}\right|}, \quad 1 \leq i \leq d .
$$

LEMMA 4.1. Let $I_{0}=[0, \xi]$ and $\tau=\xi+|D(Q) M| / r, \sigma=q_{0}^{d / 2} \xi-$ $|D(Q) M| / r$. Then

$$
\operatorname{vol} H(r) \ll_{d}(b-a) q_{0}^{-d / 2} q^{(d-2) / 2} \tau^{d-2} r^{d-2} .
$$

If $\sigma>0$ and $|a|+|b| \leq \sigma^{2} r^{2} / 5$ then

$$
\operatorname{vol} H(r) \gg_{d}(b-a) q^{-d / 2} \sigma^{d-2} r^{d-2} \text {. }
$$

Proof. See [BG99, p. 1023, Lemma 8.2], or [Els06, p. 24, Lemma 2.4.3].

Lemma 4.2. Let $I_{0}=[1-\delta, 1+\delta], 0 \leq \delta \leq 1 / 4$. Assume that $r$ is so large that

$$
\varepsilon_{1}:=r^{-1}|D(Q) M| \leq q_{0}^{1 / 2} / 4, \quad \varepsilon_{2}:=r^{-2}(|a|+|b|) \leq q_{0} / 8 .
$$

Then

$$
\operatorname{vol} H(r) \ll_{d}(b-a)\left(\delta+q_{0}^{-1 / 2} \varepsilon_{1}+2 q_{0}^{-1 / 2} \varepsilon_{2}\right) r^{d-2} q_{0}^{-d / 2} q^{(d-2) / 2} .
$$

Proof. See [BG99, p. 1025, Lemma 8.3], or [Els06, p. 26, Lemma 2.4.4].

Since $a_{r}$ defined in (3.51) satisfies

$$
\left|a_{r}\right| / r^{2} \leq q
$$

for $r$ large enough, in the case $a=a_{r}$ we obtain the following lemma by slightly modifying the proof of Lemma 4.1 given in [BG99] resp. [Els06]. Using these modifications we also get an analogue of Lemma 4.2.

LemmA 4.3. Let $I_{r}:=\left[a_{r}, b\right]$ and let $F(r):=H\left(r, I_{0}, I_{r}, M\right)$ be defined as in (4.1). There exist constants $C_{Q, 1}, C_{Q, 2}, C_{Q, 3} \geq 1$ depending on $d$ and $Q$ only and a constant $r_{0}=r_{0}(Q, M, b) \geq 1$ such that for $r \geq r_{0}$ :

(1) $\operatorname{vol} F(r) \leq\left(b-a_{r}\right) C_{Q, 1} \xi^{d-2} r^{d-2}$ for $I_{0}=[0, \xi]$.

(2) $\operatorname{vol} F(r) \geq\left(b-a_{r}\right) C_{Q, 2} \xi^{d-2} r^{d-2}$ for $I_{0}=[0, \xi]$.

(3) $\operatorname{vol} F(r) \leq\left(b-a_{r}\right) C_{Q, 3} \delta r^{d-2}$ for $I_{0}=[1-\delta, 1+\delta], 0 \leq \delta \leq 1 / 4$.

The constants $C_{Q, 1}, C_{Q, 2}, C_{Q, 3}$ can be computed explicitly.

We want to estimate the error terms caused by the approximations of the (lattice point) volumes of the hyperbolic shell $H_{r, M}$. In the notation of (3.5)-(3.6), considering, for $\varepsilon>0$,

$$
\psi_{r, \pm \varepsilon}(x)=\exp \left\{-\frac{2}{r^{2}} Q_{+}[x]\right\} \chi_{ \pm \varepsilon}\left(\frac{x}{r}\right)
$$

and

$$
\Delta_{ \pm \varepsilon}=\left|\int_{\mathbb{R}^{d}} I_{H_{r, M}}(x) \psi_{r, \pm \varepsilon}(x) d x-\sum_{x \in \mathbb{Z}^{d}} I_{H_{r, M}}(x) \psi_{r, \pm \varepsilon}(x)\right|
$$


(see (3.9) and (3.11)), we define additionally

$$
v_{\varepsilon}:=\operatorname{vol}\left(H_{r, M} \cap\left\{x \in \mathbb{R}^{d}: r(1-\varepsilon) \leq|x|_{\infty} \leq r(1+\varepsilon)\right\}\right)
$$

and get the following estimate:

Lemma 4.4. For $0<\varepsilon \leq 1 / 4$ there exists a constant $c=c(d)>0$ such that

$$
\begin{aligned}
\left|\operatorname{vol}_{\mathbb{Z}} H_{r, M}-\operatorname{vol} H_{r, M}\right| \leq \max \left\{\Delta_{-\varepsilon}, \Delta_{\varepsilon}\right\}+c(b-a) q_{0}^{-d / 2} q^{(d-2) / 2} \\
\times\left(\varepsilon+q_{0}^{-1 / 2} q^{1 / 2}|M| r^{-1}+2 q_{0}^{-1 / 2}(|a|+|b|) r^{-2}\right) r^{d-2} .
\end{aligned}
$$

Proof. Obviously, we can estimate

$\operatorname{vol}_{\mathbb{Z}} H_{r, M} \leq \sum_{x \in \mathbb{Z}^{d}} I_{H_{r, M}}(x) \psi_{r, \varepsilon}(x), \quad \operatorname{vol} H_{r, M} \leq \int_{\mathbb{R}^{d}} I_{H_{r, M}}(x) \psi_{r,-\varepsilon}(x) d x+v_{\varepsilon}$, $\operatorname{vol}_{\mathbb{Z}} H_{r, M} \geq \sum_{x \in \mathbb{Z}^{d}} I_{H_{r, M}}(x) \psi_{r,-\varepsilon}(x), \quad \operatorname{vol} H_{r, M} \geq \int_{\mathbb{R}^{d}} I_{H_{r, M}}(x) \psi_{r, \varepsilon}(x) d x-v_{\varepsilon}$.

If $\operatorname{vol}_{\mathbb{Z}} H_{r, M}-\operatorname{vol} H_{r, M} \geq 0$ these estimates imply

$$
\left|\operatorname{vol}_{\mathbb{Z}} H_{r, M}-\operatorname{vol} H_{r, M}\right| \leq \Delta_{+\varepsilon}+v_{\varepsilon},
$$

and otherwise we obtain

$$
\left|\operatorname{vol}_{\mathbb{Z}} H_{r, M}-\operatorname{vol} H_{r, M}\right| \leq \Delta_{-\varepsilon}+v_{\varepsilon} .
$$

Using Lemma 4.2 for $I_{0}=[1-\varepsilon, 1+\varepsilon]$, since $|D(Q) M| \leq q^{1 / 2}|M|$ we get $v_{\varepsilon} \ll_{d}(b-a)\left(\varepsilon+q_{0}^{-1 / 2} q^{1 / 2}|M| r^{-1}+2 q_{0}^{-1 / 2}(|a|+|b|) r^{-2}\right) r^{d-2} q_{0}^{-d / 2} q^{(d-2) / 2}$, which proves (4.5).

Lemma 4.5. Fix $a, b \in \mathbb{R}, w>0$ and let $g_{a, b, w}$ be defined as in (3.3). Then:

(1) There exist $a^{\prime} \in[a-w, a+w]$ and $b^{\prime} \in[b-w, b+w]$ such that

$$
\sum_{x \in \mathbb{Z}^{d}}\left(I_{[a, b]}-g_{a^{\prime}, b^{\prime}, w}\right)(Q[x-M]) \psi_{r, \pm \varepsilon}(x)=0 .
$$

(2) $\sup _{\substack{a^{\prime} \in[a-w, a+w] \\ b^{\prime} \in[b-w, b+w]}}\left|\int_{\mathbb{R}^{d}}\left(I_{[a, b]}-g_{a^{\prime}, b^{\prime}, w}\right)(Q[x-M]) \psi_{r, \pm \varepsilon}(x) d x\right|$

$$
\ll_{d} 8 w q_{0}^{-d / 2} q^{(d-2) / 2}\left(1+\varepsilon+q^{1 / 2} \frac{|M|}{r}\right)^{d-2} r^{d-2} .
$$

Proof. The sum in (1) is finite, since $\psi_{r, \pm \varepsilon}$ has bounded support. Hence, the map $G:\left(a^{\prime}, b^{\prime}\right) \mapsto \sum_{x \in \mathbb{Z}^{d}}\left(I_{[a, b]}-g_{a^{\prime}, b^{\prime}, w}\right)(Q[x-M]) \psi_{r, \pm \varepsilon}(x)$ is continuous and (1) follows by the intermediate value theorem.

For all $a^{\prime} \in[a-w, a+w]$ and all $b^{\prime} \in[b-w, b+w]$ we can estimate

$$
\left|\left(I_{[a, b]}-g_{a^{\prime}, b^{\prime}, w}\right)(Q[x-M])\right| \leq I_{[a-2 w, a+2 w] \cup[b-2 w, b+2 w]}(Q[x-M]) .
$$


This implies

$$
\begin{aligned}
& \sup _{a^{\prime}, b^{\prime}}\left|\int_{\mathbb{R}^{d}}\left(I_{[a, b]}-g_{a^{\prime}, b^{\prime}, w}\right)(Q[x-M]) \psi_{r, \pm \varepsilon}(x) d x\right| \\
& \quad \leq \int I_{[a-2 w, a+2 w] \cup[b-2 w, b+2 w]}(Q[x-M]) \psi_{r, \pm \varepsilon}(x) d x \\
& \quad \leq \int\left(I_{[a-2 w, a+2 w]}+I_{[b-2 w, b+2 w]}\right)(Q[x-M]) I_{[0, r(1+\varepsilon)]}\left(|x|_{\infty}\right) d x,
\end{aligned}
$$

since $\psi_{r, \pm \varepsilon}(x) \leq I_{[0, r(1+\varepsilon)]}\left(|x|_{\infty}\right)$.

Using Lemma 4.1 with $I_{0}=[0,1+\varepsilon]$, by $(4.7)$ we get

$$
\begin{aligned}
\sup _{a^{\prime}, b^{\prime}} \mid \int_{\mathbb{R}^{d}}\left(I_{[a, b]}-\right. & \left.g_{a^{\prime}, b^{\prime}, w}\right)(Q[x-M]) \psi_{r, \pm \varepsilon}(x) d x \mid \\
& \ll_{d} 8 w q_{0}^{-d / 2} q^{(d-2) / 2}\left(1+\varepsilon+r^{-1}|D(Q) M|\right)^{d-2} r^{d-2} \\
& \leq 8 w q_{0}^{-d / 2} q^{(d-2) / 2}\left(1+\varepsilon+r^{-1} q^{1 / 2}|M|\right)^{d-2} r^{d-2}
\end{aligned}
$$

which proves $(2)$.

Lemma 4.6. Consider $\Delta_{ \pm \varepsilon}, \varepsilon>0$, defined in (3.11). Then

$$
\begin{aligned}
\Delta_{ \pm \varepsilon} \ll_{d} \sup _{\substack{a^{\prime} \in[a-w, a+w] \\
b^{\prime} \in[b-w, b+w]}}\left|V_{w, \pm \varepsilon}^{\mathbb{R}}\left(r ; a^{\prime}, b^{\prime}, M\right)-V_{w, \pm \varepsilon}^{\mathbb{Z}}\left(r ; a^{\prime}, b^{\prime}, M\right)\right| \\
+8 w q_{0}^{-d / 2} q^{(d-2) / 2}\left(1+\varepsilon+q^{1 / 2} \frac{|M|}{r}\right)^{d-2} r^{d-2},
\end{aligned}
$$

where $V_{w, \pm \varepsilon}^{\mathbb{R}}\left(r ; a^{\prime}, b^{\prime}, M\right)$ and $V_{w, \pm \varepsilon}^{\mathbb{Z}}\left(r ; a^{\prime}, b^{\prime}, M\right)$ are defined as in (3.6) and (3.5) respectively.

Proof. Using functions $g_{a, b, w}$ defined in (3.3) by the triangle inequality (recall the definition of $\psi_{r, \pm \varepsilon}$ in (3.9)) we obtain

$$
\begin{aligned}
\Delta_{ \pm \varepsilon}= & \left|\int_{\mathbb{R}^{d}} I_{H_{r, M}}(x) \psi_{r, \pm \varepsilon}(x) d x-\sum_{x \in \mathbb{Z}^{d}} I_{H_{r, M}}(x) \psi_{r, \pm \varepsilon}(x)\right| \\
\leq & \left|\int_{\mathbb{R}^{d}}\left(I_{[a, b]}-g_{a^{\prime}, b^{\prime}, w}\right)(Q[x-M]) \psi_{r, \pm \varepsilon}(x) d x\right| \\
& +\left|V_{w, \pm \varepsilon}^{\mathbb{R}}\left(r ; a^{\prime}, b^{\prime}, M\right)-V_{w, \pm \varepsilon}^{\mathbb{Z}}\left(r ; a^{\prime}, b^{\prime}, M\right)\right| \\
& +\left|\sum_{x \in \mathbb{Z}^{d}}\left(I_{[a, b]}-g_{a^{\prime}, b^{\prime}, w}\right)(Q[x-M]) \psi_{r, \pm \varepsilon}(x)\right| .
\end{aligned}
$$


Choosing $a^{\prime}, b^{\prime}$ according to Lemma 4.5(1) and estimating the first summand by taking the supremum, we obtain

$$
\begin{aligned}
\Delta_{ \pm \varepsilon} \leq & \sup _{\substack{a^{\prime} \in[a-w, a+w] \\
b^{\prime} \in[b-w, b+w]}}\left|\int_{\mathbb{R}^{d}}\left(I_{[a, b]}-g_{a^{\prime}, b^{\prime}, w}\right)(Q[x-M]) \psi_{r, \pm \varepsilon}(x) d x\right| \\
& +\sup _{\substack{a^{\prime} \in[a-w, a+w] \\
b^{\prime} \in[b-w, b+w]}}\left|V_{w, \pm \varepsilon}^{\mathbb{R}}\left(r ; a^{\prime}, b^{\prime}, M\right)-V_{w, \pm \varepsilon}^{\mathbb{Z}}\left(r ; a^{\prime}, b^{\prime}, M\right)\right| .
\end{aligned}
$$

The application of Lemma 4.5(2) completes the proof.

Repeating the proofs of Lemmas 4.4-4.6 in the case $a=a_{r}$ with the use of Lemma 4.3 instead of Lemmas 4.1 and 4.2 we get immediately

Lemma 4.7. Let $F_{r, M}(b)$ be defined as in (3.52). Then there exist constants $r_{0}=r_{0}(Q, M, b) \geq 1$ and $c_{Q, 1}, c_{Q, 2} \geq 1$ depending on $Q$ and $d$ only such that for $w>0$ and $0<\varepsilon<1 / 4$,

$$
\begin{aligned}
\left|\operatorname{vol}_{\mathbb{Z}} F_{r, M}(b)-\operatorname{vol} F_{r, M}(b)\right| \leq\left(c_{Q, 1}\left(b-a_{r}\right) \varepsilon+c_{Q, 2} w(1+\varepsilon)^{d-2}\right) r^{d-2} & \\
& +\sup _{\substack{a^{\prime} \in\left[a_{r}-w, a_{r}+w\right] \\
b^{\prime} \in[b-w, b+w]}}\left|V_{w, \pm \varepsilon}^{\mathbb{R}}\left(r ; a^{\prime}, b^{\prime}, M\right)-V_{w, \pm \varepsilon}^{\mathbb{Z}}\left(r ; a^{\prime}, b^{\prime}, M\right)\right|,
\end{aligned}
$$

where $V_{w, \pm \varepsilon}^{\mathbb{R}}\left(r ; a^{\prime}, b^{\prime}, M\right)$ and $V_{w, \pm \varepsilon}^{\mathbb{Z}}\left(r ; a^{\prime}, b^{\prime}, M\right)$ are defined as in (3.6) and (3.5) respectively.

Lemma 4.8. For any $\beta>0$ and $T \in \mathbb{R}$,

$$
\frac{1}{2 \pi i} \int_{\beta-i \infty}^{\beta+i \infty} \exp \{z T\} \frac{d z}{z^{2}}=\max \{T, 0\}=T_{+} .
$$

Proof. Complement the interval $(\beta-i \infty, \beta+i \infty)$ by an infinite half circle in $\operatorname{Re} z \geq 0$ (resp. $\operatorname{Re} z \leq 0$ ) for $T<0$ (resp. $T \geq 0$ ) and apply standard residue calculus.

Lemma 4.9. Let $\Omega$ be a symmetric, $d \times d$ complex matrix whose imaginary part is positive definite. Then

$$
\begin{aligned}
\sum_{m \in \mathbb{Z}^{d}} \exp \{\pi i \Omega[m]+2 \pi i\langle m, v\rangle\}= & (\operatorname{det}(\Omega / i))^{-1 / 2} \exp \left\{-\pi i \Omega^{-1}[v]\right\} \\
& \times \sum_{n \in \mathbb{Z}^{d}} \exp \left\{-\pi i \Omega^{-1}[n]+2 \pi i\left\langle n, \Omega^{-1} v\right\rangle\right\}
\end{aligned}
$$

and

$$
\int_{\mathbb{R}^{d}} \exp \{\pi i \Omega[x]+2 \pi i\langle x, v\rangle\} d x=(\operatorname{det}(\Omega / i))^{-1 / 2} \exp \left\{-\pi i \Omega^{-1}[v]\right\},
$$


where $\Omega^{-1}[x]$ denotes the quadratic form $\left\langle\Omega^{-1} x, x\right\rangle$, defined by the inverse operator $\Omega^{-1}: \mathbb{C}^{d} \rightarrow \mathbb{C}^{d}$ (which exists since $\Omega$ is an element of Siegel's upper half plane).

Proof. See [Mum83, p. 195 (5.6) and Lemma 5.8].

Corollary 4.10. Let $z \in \mathbb{C}^{d}$ with $\operatorname{Re} z>0, v \in \mathbb{C}^{d}$, and let $\Omega$ be a positive definite, symmetric $d \times d$ matrix. Then

$$
\begin{aligned}
\sum_{m \in \mathbb{Z}^{d}} \exp \{-z \Omega[m] & +2 \pi i\langle m, v\rangle\} \\
& =\left(\operatorname{det}\left(z \frac{\Omega}{\pi}\right)\right)^{-1 / 2} \sum_{n \in \mathbb{Z}^{d}} \exp \left\{-\frac{\pi^{2}}{z} \Omega^{-1}[n+v]\right\} .
\end{aligned}
$$

Proof. Apply Lemma 4.9 to the matrix $(i / \pi) z \Omega$.

Lemma 4.11. Let $z=1 / r^{2}+i t, r>0, t \in \mathbb{R}$ and $v \in \mathbb{C}^{d}$. Then

$$
\sum_{m \in \mathbb{Z}^{d}} \exp \left\{-\frac{2}{r^{2}} Q_{+}[m]-z Q[m]+2 \pi i\langle m, v\rangle\right\}
$$

$$
\begin{aligned}
= & \operatorname{det}\left(\frac{1}{\pi}\left(\frac{2}{r^{2}} Q_{+}+z Q\right)\right)^{-1 / 2} \exp \left\{-\pi^{2}\left(\frac{2}{r^{2}} Q_{+}+z Q\right)^{-1}[v]\right\} \\
& \times \sum_{n \in \mathbb{Z}^{d}} \exp \left\{-\pi^{2}\left(\frac{2}{r^{2}} Q_{+}+z Q\right)^{-1}[n]-2 \pi^{2}\left\langle\left(\frac{2}{r^{2}} Q_{+}+z Q\right)^{-1} n, v\right\rangle\right\},
\end{aligned}
$$

and

$$
\begin{aligned}
& \int_{\mathbb{R}^{d}} \exp \left\{-\frac{2}{r^{2}} Q_{+}[x]-z Q[x]+2 \pi i\langle x, v\rangle\right\} d x \\
& \quad=\operatorname{det}\left(\frac{1}{\pi}\left(\frac{2}{r^{2}} Q_{+}+z Q\right)\right)^{-1 / 2} \exp \left\{-\pi^{2}\left(\frac{2}{r^{2}} Q_{+}+z Q\right)^{-1}[v]\right\},
\end{aligned}
$$

where $\left(\frac{2}{r^{2}} Q_{+}+z Q\right)^{-1}[x]$ denotes the quadratic form $\left\langle\left(\frac{2}{r^{2}} Q_{+}+z Q\right)^{-1} x, x\right\rangle$, defined by means of the positive definite operator $\left(\frac{2}{r^{2}} Q_{+}+z Q\right)^{-1}: \mathbb{R}^{d} \rightarrow \mathbb{R}^{d}$.

Proof. For $\Omega:=\frac{i}{\pi}\left(\frac{2}{r^{2}} Q_{+}+z Q\right)$ and $z=1 / r^{2}+i t, t \in \mathbb{R}$, the imaginary part $\operatorname{Im} \Omega$ is positive definite. The application of Lemma 4.9 to $\Omega$ completes the proof.

LEMma 4.12. Let $\theta_{v}(z)$ and $\theta_{0, v}(z)$ denote the theta sum and theta integral in (3.16) and (3.17) respectively. Then there is a constant $c=c(Q)>0$ such that for $r \geq r_{0}=r_{0}(Q, M) \geq 1$ and $t \in \mathbb{R}$ with $|t|<1 / r$,

$$
\begin{gathered}
\left|\left(\theta_{v}-\theta_{0, v}\right)\left(r^{-2}+i t\right)\right| \ll_{d} q_{0}^{-d / 2}\left|r^{-2}+i t\right|^{-d / 2} \exp \left\{-c \operatorname{Re}\left(\left(r^{-2}+i t\right)^{-1}\right)\right\} \\
+2 I_{(r, \infty)}(|v|) .
\end{gathered}
$$


Proof. Using Lemma 4.11, by (3.16), (3.17) and the self-adjointness of the matrix $\left(\frac{2}{r^{2}} Q_{+} z Q\right)^{-1}$ we obtain

$$
\begin{aligned}
\left(\theta_{v}-\theta_{0, v}\right)(z) & =\exp \{-z Q[M]\} \operatorname{det}\left(\frac{1}{\pi} \Omega\right)^{-1 / 2} \exp \left\{-\pi^{2} \Omega^{-1}\left[-\frac{\tilde{v}}{2 \pi r}\right]\right\} \\
& \times \sum_{n \in \mathbb{Z}^{d} \backslash\{0\}} \exp \left\{-\pi^{2} \Omega^{-1}[n]-2 \pi^{2}\left\langle\Omega^{-1} n,-\frac{\tilde{v}}{2 \pi r}\right\rangle\right\} \\
= & \exp \{-z Q[M]\} \operatorname{det}\left(\frac{1}{\pi} \Omega\right)^{-1 / 2} \sum_{n \in \mathbb{Z}^{d} \backslash\{0\}} \exp \left\{-\Omega^{-1}\left[\pi n-\frac{\tilde{v}}{2 r}\right]\right\},
\end{aligned}
$$

where $\Omega:=\frac{2}{r^{2}} Q_{+}+z Q$ and $\tilde{v}:=2 r \operatorname{Im}(z) Q M-v$. Note that for $z=$ $r^{-2}+i t$ and $t \leq 1 / r$ there exists a constant $c_{0}=c_{0}(Q, M)>0$ such that $|2 r \operatorname{Im}(z) Q M| \leq c_{0}$ uniformly in $r$. Using

$\operatorname{det}\left(\frac{1}{\pi} \Omega\right)=\frac{1}{\pi^{d}} \prod_{1 \leq j \leq d}\left(\frac{2}{r^{2}}\left|q_{j}\right|+z q_{j}\right) \quad$ and $\quad\left|\frac{2}{r^{2}}\right| q_{j}\left|+z q_{j}\right| \geq\left|q_{j}\right| \cdot\left|r^{-2}+i t\right|$ for $z=r^{-2}+i t$ and all $1 \leq j \leq d$, we have

$$
\left|\operatorname{det}\left(\frac{1}{\pi} \Omega\right)^{-1 / 2}\right| \leq \pi^{d / 2} q_{0}^{-d / 2}|z|^{-d / 2}
$$

Since $\Omega$ can be orthogonal diagonalized, the matrix $\operatorname{Re}\left(\Omega^{-1}\right)$ has eigenvalues $\operatorname{Re}\left(\left(\frac{2}{r^{2}}\left|q_{j}\right|+z q_{j}\right)^{-1}\right), 1 \leq j \leq d$. For $t \leq 1 / r$ we have

$$
\operatorname{Re}\left(\left(\frac{2}{r^{2}}\left|q_{j}\right|+z q_{j}\right)^{-1}\right) \geq \frac{1}{\left|q_{j}\right|} \operatorname{Re}\left(z^{-1}\right) \geq \frac{1}{q} \operatorname{Re}\left(z^{-1}\right), \quad 1 \leq j \leq d .
$$

Hence,

$$
\begin{aligned}
\left|\exp \left\{-\pi^{2} \Omega^{-1}\left[n-\frac{\tilde{v}}{2 \pi r}\right]\right\}\right| & =\exp \left\{-\operatorname{Re}\left(\Omega^{-1}\left[\pi n-\frac{\tilde{v}}{2 r}\right]\right)\right\} \\
& =\exp \left\{-\operatorname{Re}\left(\Omega^{-1}\right)\left[\pi n-\frac{\tilde{v}}{2 r}\right]\right\} \\
& \leq \exp \left\{-\frac{1}{q} \operatorname{Re}\left(z^{-1}\right)\left|\pi n-\frac{\tilde{v}}{2 r}\right|^{2}\right\}
\end{aligned}
$$


Using (4.14)-(4.16) we get

$$
\begin{aligned}
\left|\left(\theta_{v}-\theta_{0, v}\right)\left(r^{-2}+i t\right)\right| \ll_{d} \exp \left\{-\frac{1}{r^{2}} Q[M]\right\} q_{0}^{-d / 2}\left|r^{-2}+i t\right|^{-d / 2} \\
\times \sum_{n \in \mathbb{Z}^{d} \backslash\{0\}} \exp \left\{-\frac{1}{q} \operatorname{Re}\left(\left(r^{-2}+i t\right)^{-1}\right)\left|\pi n-\frac{\tilde{v}}{2 r}\right|^{2}\right\} .
\end{aligned}
$$

For $|\tilde{v}| \leq \pi r$ we obtain

$$
\exp \left\{-\frac{1}{q} \operatorname{Re}\left(\left(r^{-2}+i t\right)^{-1}\right)\left|\pi n-\frac{\tilde{v}}{2 r}\right|^{2}\right\} \leq \exp \left\{-\frac{1}{q} \operatorname{Re}\left(\left(r^{-2}+i t\right)^{-1}\right) \frac{|\pi n|^{2}}{2}\right\}
$$

and hence, for an appropriate constant $c=c(Q)>0$,

$$
\begin{aligned}
\sum_{n \in \mathbb{Z}^{d} \backslash\{0\}} \exp \left\{-\frac{1}{q}\right. & \left.\operatorname{Re}\left(\left(r^{-2}+i t\right)^{-1}\right)\left|\pi n-\frac{\tilde{v}}{2 r}\right|^{2}\right\} \\
\leq & \sum_{n \in \mathbb{Z}^{d} \backslash\{0\}} \exp \left\{-\frac{1}{q} \operatorname{Re}\left(\left(r^{-2}+i t\right)^{-1}\right) \frac{|\pi n|^{2}}{2}\right\} \\
& \ll \exp \left\{-c \operatorname{Re}\left(\left(r^{-2}+i t\right)^{-1}\right)\right\} .
\end{aligned}
$$

For $|\tilde{v}|>\pi r$ set $\tilde{v}=L \pi r+w$ with $L \in \mathbb{Z},|w| \leq \pi r$. Then $w=\widetilde{v^{\prime}}$ for $v^{\prime}:=v+L \pi r$. By (4.14) we have obviously $\theta_{v}=\theta_{v^{\prime}}$, and therefore by (4.18) and (4.17) we get

$$
\begin{aligned}
& \left|\left(\theta_{v}-\theta_{0, v}\right)\left(r^{-2}+i t\right)\right| \\
\leq & \left|\left(\theta_{v^{\prime}}-\theta_{0, v^{\prime}}\right)\left(r^{-2}+i t\right)\right|+\left|\left(\theta_{0, v^{\prime}}-\theta_{0, v}\right)\left(r^{-2}+i t\right)\right| \\
\ll_{d} & \exp \left\{-\frac{1}{r^{2}} Q[M]\right\} q_{0}^{-d / 2}\left|r^{-2}+i t\right|^{-d / 2} \exp \left\{-c \operatorname{Re}\left(\left(r^{-2}+i t\right)^{-1}\right)\right\} \\
& +\left|\theta_{0, v^{\prime}}\left(r^{-2}+i t\right)\right|+\left|\theta_{0, v}\left(r^{-2}+i t\right)\right| \\
\leq & \exp \left\{-\frac{1}{r^{2}} Q[M]\right\} q_{0}^{-d / 2}\left|r^{-2}+i t\right|^{-d / 2} \exp \left\{-c \operatorname{Re}\left(\left(r^{-2}+i t\right)^{-1}\right)\right\}+2 .
\end{aligned}
$$

The result now follows by (4.17)-(4.19) for $r \geq r_{0}, r_{0} \geq 1$ large enough, since $|\tilde{v}|>\pi r$ implies $|v| \geq \pi r-c_{0}(Q, M) \geq r$ for $r$ large enough.

LEMMA 4.13. Let $\theta_{v}(z)$ denote the theta function in (3.16) depending on $Q$ and $v \in \mathbb{C}^{d}$. For $r \geq r_{0}=r_{0}(Q, M) \geq 1$ and $t \in \mathbb{R}$,

$$
\left|\theta_{v}\left(r^{-2}+i t\right)\right| \ll_{d}(\operatorname{det} \Omega)^{-1 / 4} r^{d / 2} \psi(r, t)^{1 / 2},
$$

where

$$
\psi(r, t)=\sum_{m, n \in \mathbb{Z}^{d}} \exp \left\{-\frac{r^{2}}{2} \Omega^{-1}[\pi m-2 t Q n]-\frac{2}{r^{2}} \Omega[n]\right\},
$$

with $\Omega:=2 Q_{+}+Q$. 
Note that the right hand side of this inequality is independent of $v \in \mathbb{C}^{d}$.

Proof. For any $x, y \in \mathbb{R}^{d}$ we have

$$
\begin{aligned}
2(\Omega[x]+\Omega[y]) & =\Omega[x+y]+\Omega[x-y] \\
\langle\Omega(x+y), x-y\rangle & =\Omega[x]-Q[y] .
\end{aligned}
$$

Rearranging $\theta_{v}(z) \overline{\theta_{v}(z)}$ and using (4.22), we would like to use $m+n$ and $m-n$ as new summation variables on a lattice. But both vectors have the same parity, i.e., $m+n \equiv m-n \bmod 2$. Since they are dependent one has to consider the $2^{d}$ sublattices indexed by $\alpha=\left(\alpha_{1}, \ldots, \alpha_{d}\right)$ with $\alpha_{j}=0,1$, for $1 \leq j \leq d$ :

$$
\mathbb{Z}_{\alpha}^{d}:=\left\{m \in \mathbb{Z}^{d}: m \equiv \alpha \bmod 2\right\},
$$

where, for $m=\left(m_{1}, \ldots, m_{d}\right), m \equiv \alpha \bmod 2$ means $m_{j} \equiv \alpha_{j} \bmod 2$ for $1 \leq j \leq d$. Thus writing

$$
\theta_{v, \alpha}(z):=\sum_{m \in \mathbb{Z}_{\alpha}^{d}} \exp \left\{-\frac{2}{r^{2}} Q_{+}[m]-z Q[m]-i\left\langle m, \frac{v}{r}-2 t Q M\right\rangle\right\},
$$

we obtain $\theta_{v}(z)=\exp \{-z Q[M]\} \sum_{\alpha} \theta_{v, \alpha}(z)$ and hence by the CauchySchwarz inequality

$$
\left|\theta_{v}(z)\right|^{2} \leq 2^{d} \exp \left\{-\frac{2}{r^{2}} Q[M]\right\} \sum_{\alpha}\left|\theta_{v, \alpha}(z)\right|^{2} .
$$

Using (4.22) and the absolute convergence of $\theta_{\alpha}(z)$, we may rewrite the quantity $\theta_{v, \alpha}(z) \overline{\theta_{v, \alpha}(z)}$ for $z=1 / r^{2}+i t$ and $\tilde{v}:=v-2 \operatorname{tr} Q M$ as

$$
\begin{aligned}
& \theta_{v, \alpha}(z) \overline{\theta_{v, \alpha}(z)} \\
= & \sum_{m, n \in \mathbb{Z}_{\alpha}^{d}} \exp \left\{-\frac{1}{r^{2}}(\Omega[m]+\Omega[n])-i t(Q[m]-Q[n])-i\left\langle m-n, \frac{\tilde{v}}{r}\right\rangle\right\} \\
= & \sum_{m, n \in \mathbb{Z}_{\alpha}^{d}} \exp \left\{-\frac{2}{r^{2}}(\Omega[\bar{m}]+\Omega[\bar{n}])-2 i\left\langle 2 t Q \bar{m}+\frac{\tilde{v}}{r}, \bar{n}\right\rangle\right\}
\end{aligned}
$$

where $\bar{m}=(m+n) / 2$ and $\bar{n}=(m-n) / 2$. Note that the map

$$
H: \bigcup_{\alpha} \mathbb{Z}_{\alpha}^{d} \times \mathbb{Z}_{\alpha}^{d} \rightarrow \mathbb{Z}^{d} \times \mathbb{Z}^{d}, \quad(m, n) \mapsto((m+n) / 2,(m-n) / 2),
$$


is a bijection. Therefore by (4.23) we get

$$
\begin{aligned}
& \exp \left\{\frac{2}{r^{2}} Q[M]\right\}\left|\theta_{v}(z)\right|^{2} \\
& \ll_{d} \sum_{\alpha \in\{0,1\}^{d}} \sum_{\bar{m}, \bar{n} \in \mathbb{Z}_{\alpha}^{d}} \exp \left\{-\frac{2}{r^{2}}(\Omega[\bar{m}]+\Omega[\bar{n}])-2 i\left\langle 2 t Q \bar{m}+\frac{\tilde{v}}{r}, \bar{n}\right\rangle\right\} \\
& =\sum_{\bar{m}, \bar{n} \in \mathbb{Z}^{d}} \exp \left\{-\frac{2}{r^{2}}(\Omega[\bar{m}]+\Omega[\bar{n}])-2 i\left\langle 2 t Q \bar{m}+\frac{\tilde{v}}{r}, \bar{n}\right\rangle\right\} .
\end{aligned}
$$

In this double sum fix $\bar{n}$ and sum over $\bar{m} \in \mathbb{Z}^{d}$ first. Using Corollary 4.10 for $z=2 / r^{2}$, we get for $\delta:=\left(\operatorname{det}\left(\frac{2}{\pi r^{2}} \Omega\right)\right)^{-1 / 2}$, by the symmetry of $Q$,

$$
\begin{aligned}
\theta_{v}(z, \bar{n}) & :=\sum_{\bar{m} \in \mathbb{Z}^{d}} \exp \left\{-\frac{2}{r^{2}}(\Omega[\bar{m}]+\Omega[\bar{n}])-2 i\left\langle 2 t Q \bar{m}+\frac{\tilde{v}}{r}, \bar{n}\right\rangle\right\} \\
& =\delta \sum_{m \in \mathbb{Z}^{d}} \exp \left\{-\frac{r^{2}}{2} \Omega^{-1}[\pi m-2 t Q \bar{n}]-\frac{2}{r^{2}} \Omega[\bar{n}]-2 i\left\langle\frac{\tilde{v}}{r}, \bar{n}\right\rangle\right\} .
\end{aligned}
$$

Thus,

$$
\left|\theta_{v}(z, \bar{n})\right| \leq \delta \sum_{m \in \mathbb{Z}^{d}} \exp \left\{-\frac{r^{2}}{2} \Omega^{-1}[\pi m-2 t Q \bar{n}]-\frac{2}{r^{2}} \Omega[\bar{n}]\right\} .
$$

Hence, by (4.25) and (4.26) we obtain

$$
\begin{aligned}
\left|\theta_{v}(z)\right|^{2} \ll_{d} & \exp \left\{-\frac{2}{r^{2}} Q[M]\right\}(\operatorname{det} \Omega)^{-1 / 2} r^{d} \\
& \times \sum_{m, \bar{n} \in \mathbb{Z}^{d}} \exp \left\{-\frac{r^{2}}{2} \Omega^{-1}[\pi m-2 t Q \bar{n}]-\frac{2}{r^{2}} \Omega[\bar{n}]\right\},
\end{aligned}
$$

which proves Lemma 4.13 for $r>r_{0}=r_{0}(Q, M):=|Q[M]|^{1 / 2}+1$.

In the following we shall use some facts from the geometry of numbers (see [Dav58]).

Let $F: \mathbb{R}^{d} \rightarrow[0, \infty)$ denote a norm on $\mathbb{R}^{d}$, that is, $F(\alpha x)=|\alpha| F(x)$ for $\alpha \in \mathbb{R}$, and $F(x+y) \leq F(x)+F(y)$. The successive minima $M_{1} \leq$ $\cdots \leq M_{d}$ of $F$ with respect to the lattice $\mathbb{Z}^{d}$ are defined as follows: Let $M_{1}=\inf \left\{F(m): m \neq 0, m \in \mathbb{Z}^{d}\right\}$ and define $M_{k}$ as the infimum of $\lambda>0$ such that the set $\left\{m \in \mathbb{Z}^{d}: F(m)<\lambda\right\}$ contains $k$ linearly independent vectors. It is easy to see that these infima are attained, that is, there exist linearly independent vectors $a_{1}, \ldots, a_{d} \in \mathbb{Z}^{d}$ such that $F\left(a_{j}\right)=M_{j}$.

LEMMA 4.14. Let $L_{j}(x)=\sum_{k=1}^{d} q_{j k} x_{k}, 1 \leq j \leq d$, denote linear forms on $\mathbb{R}^{d}$ such that $q_{j k}=q_{k j}, j, k=1, \ldots, d$. Assume that $r \geq 1$ and let $\|v\|$ 
denote the distance of the number $v$ to the nearest integer. Then the number of $m=\left(m_{1}, \ldots, m_{d}\right) \in \mathbb{Z}^{d}$ such that

$$
\left\|L_{j}(m)\right\|<r^{-1}, \quad\left|m_{j}\right|<r, \quad \text { for all } 1 \leq j \leq d,
$$

is bounded from above by $c_{d}\left(M_{1} \cdots M_{d}\right)^{-1}$, where $c_{d}>0$ denotes a constant depending on $d$ only, $M_{1} \leq \cdots \leq M_{d}$ are the first $d$ of the $2 d$ successive minima $M_{1} \leq \cdots \leq M_{2 d}$ of the norm $F: \mathbb{R}^{2 d} \rightarrow[0, \infty)$ defined for $y=$ $(x, \bar{x}) \in \mathbb{R}^{2 d}, x, \bar{x} \in \mathbb{R}^{d}, \bar{x}=\left(\bar{x}_{1}, \ldots, \bar{x}_{d}\right)$, as

$$
F(y):=\max \left\{r\left|L_{1}(x)-\bar{x}_{1}\right|, \ldots, r\left|L_{d}(x)-\bar{x}_{d}\right|, r^{-1}|x|_{\infty}\right\} .
$$

Moreover,

$$
\frac{1}{2 d} \leq M_{k} M_{2 d+1-k} \leq(2 d)^{2 d-1}, \quad 1 \leq k \leq 2 d .
$$

Proof. See [Dav58, (20), p. 113, Lemma 3].

Note that for some constant, say $c(d)>0$, depending on $d$ only,

$$
r^{-1} \leq M_{1} \leq \cdots \leq M_{d} \leq c(d),
$$

where the first inequality is obvious by $F(m, \bar{m}) \geq r^{-1}|m|_{\infty}$. If here $m=0$ then $\bar{m} \neq 0$ and $F(m, \bar{m})=r|\bar{m}|_{\infty} \geq r^{-1}|\bar{m}|_{\infty} \geq r^{-1}$. Finally, $M_{d} \ll_{d} 1$ follows from (4.28) for $k=d$.

In the following we shall consider linear forms

$$
L_{j}(x)=\sum_{k=1}^{d} t q_{j k} x_{k}, \quad 1 \leq j \leq d,
$$

where $Q=\left(q_{i j}\right), i, j=1, \ldots, d$, and $t \in \mathbb{R}$ is arbitrary. We denote the corresponding successive minima of the norm $F(\cdot)$ defined by $(4.27)$ and (4.30) for fixed $t$ by $M_{j, t}, j=1, \ldots, d$. Thus, we can write

$$
M_{j, t}=|L(m, n, t)|_{\infty}
$$

for some $m, n \in \mathbb{Z}^{d}$, where

$$
\begin{aligned}
& L(m, n, t) \\
& \quad=\left(r\left(m_{1}-t(Q n)_{1}\right), \ldots, r\left(m_{d}-t(Q n)_{d}\right), r^{-1} n_{1}, \ldots, r^{-1} n_{d}\right) .
\end{aligned}
$$

It is easy to see from the definition that

$$
M_{j, t}=M_{j,-t}, \quad j=1, \ldots, d, t \in \mathbb{R} .
$$

Lemma 4.15. Let $r \geq 1$. Then

$$
\left|\theta\left(r^{-2}+i t \pi / 2\right)\right| \ll_{d} q_{0}^{-3 d / 4} r^{d / 2}\left(M_{1, t} \cdots M_{d, t}\right)^{-1 / 2} .
$$

Proof. By Lemma 4.13 we need to estimate the theta series $\psi(r, t \pi / 2)$. Since the matrix $\Omega=2 Q_{+}+Q$ is positive definite we may use the inequalities 
$\Omega^{-1}[x] \geq \frac{1}{3 q}|x|_{\infty}^{2}$ and $\Omega[x] \geq q_{0}|x|_{\infty}^{2}$ to get, with $c_{Q}=\min \left\{\frac{\pi^{2}}{6 q}, 2 q_{0}\right\}$,

$$
\psi(r, t \pi / 2) \ll_{d} \sum_{m, n \in \mathbb{Z}^{d}} \exp \left\{-c_{Q}|L(m, n, t)|_{\infty}^{2}\right\},
$$

where $L(m, n, t)$ is defined in (4.32). Let

$$
H:=\left\{(m, n) \in \mathbb{Z}^{2 d}:|L(m, n, t)|_{\infty}<1\right\} .
$$

Now, Lemma 4.14 may be restated for the forms (4.30) as

$$
\# H \ll_{d}\left(M_{1, t} \cdots M_{d, t}\right)^{-1} .
$$

In order to bound $\psi(r, t \pi / 2)$, for $k:=\left(k_{1}, \ldots, k_{2 d}\right) \in \mathbb{Z}^{2 d}$ we introduce the sets

$$
\begin{aligned}
& B_{k}:=\left[k_{1}-1 / 2, k_{1}+1 / 2\right) \times \cdots \times\left[k_{2 d}-1 / 2, k_{2 d}+1 / 2\right), \\
& H_{k}:=\left\{(m, n) \in \mathbb{Z}^{2 d}: L(m, n, t) \in B_{k}\right\}
\end{aligned}
$$

such that $\mathbb{R}^{2 d}=\bigcup_{k} B_{k}$. For any fixed $\left(m^{*}, n^{*}\right) \in H_{k}$ we have

$$
\left(m-m^{*}, n-n^{*}\right) \in H \quad \text { for any }(m, n) \in H_{k} .
$$

Hence, for any $k \in \mathbb{Z}^{2 d}$,

$$
\# H_{k} \leq \# H \ll_{d}\left(M_{1, t} \cdots M_{d, t}\right)^{-1} .
$$

Since $x \in B_{k}$ implies $|x|_{\infty} \geq|k|_{\infty} / 2$, by (4.34) and (4.36) we obtain

$$
\begin{aligned}
\psi(r, t \pi / 2) & \ll_{d} \# H_{0}+\sum_{k \in \mathbb{Z}^{2 d} \backslash 0} \sum_{m, n \in \mathbb{Z}^{2 d}} I_{\left\{L(m, n, t) \in B_{k}\right\}} \exp \left\{-c_{Q}|k|_{\infty}^{2} / 4\right\} \\
& \ll_{d}\left(M_{1, t} \cdots M_{d, t}\right)^{-1} \sum_{k \in \mathbb{Z}^{2 d}} \exp \left\{-c_{Q}|k|_{\infty}^{2} / 4\right\} \\
& \ll_{d}\left(M_{1, t} \cdots M_{d, t}\right)^{-1}\left(c_{Q}^{-1 / 2}+1\right)^{2 d},
\end{aligned}
$$

using bounds similar to (4.18). Some simple bounds together with Lemma 4.13 finally conclude the proof of Lemma 4.15.

In the following we consider an arbitrary, real, symmetric, non-degenerate $d^{*} \times d^{*}$ matrix $Q^{*}$. The norm on $\mathbb{R}^{d^{*}}$, associated to (4.32), and the associated successive minima are denoted by $\left|L^{*}\right|_{\infty}$ and $M_{j, t}^{*}, 1 \leq j \leq d^{*}$, respectively.

Lemma 4.16. Let $(m, n),\left(m^{\prime}, n^{\prime}\right) \in \mathbb{Z}^{2 d^{*}} \backslash 0, t, t^{\prime} \in \mathbb{R}$ and $r \geq 1$. Let $M:=\left|L^{*}(m, n, t)\right|_{\infty}$ and $M^{\prime}:=\left|L^{*}\left(m^{\prime}, n^{\prime}, t^{\prime}\right)\right|_{\infty}$. Assume that $\left\langle Q^{*} n, n^{\prime}\right\rangle>0$ and

$$
\max \left\{M, M^{\prime}\right\} \leq\left(4 d^{*}\right)^{-1 / 2}
$$

Set

$$
\Delta=\Delta\left(m, n ; m^{\prime}, n^{\prime}\right):=\left|\left\langle n^{\prime}, m\right\rangle-\left\langle m^{\prime}, n\right\rangle\right|
$$


Then

$$
\begin{aligned}
& \text { (i) } \Delta=0 \Rightarrow\left|t-t^{\prime}\right| \leq \frac{\left(d^{*}\right)^{1 / 2} \max \left\{M, M^{\prime}\right\}\left(|n|+\left|n^{\prime}\right|\right)}{r\left\langle Q^{*} n, n^{\prime}\right\rangle} \\
& \text { (ii) } \Delta \neq 0 \Rightarrow\left|t-t^{\prime}\right| \geq\left\langle Q^{*} n, n^{\prime}\right\rangle^{-1} / 2
\end{aligned}
$$

In particular, if we assume $n=n^{\prime}$ and (4.37) then alternative (i) in (4.39) holds.

Furthermore, assuming $(m, n) \in \mathbb{Z}^{2 d^{*}} \backslash 0$ and $M=\left|L^{*}(m, n, t)\right|_{\infty} \leq$ $\left(4 d^{*}\right)^{-1 / 2}$ we have either

$$
\text { (i) }|t| \leq \frac{2 d^{*} M|n|}{r\left|Q^{*} n\right|} \quad \text { or } \quad \text { (ii) }|t| \geq \frac{1}{2\left|Q^{*} n\right|} \text {. }
$$

This means $t, t^{\prime}$ resp. $t, 0$ have to be either "near" to each other or "far" apart.

Proof. See [Göt04, p. 217, Lemma 3.6] or [Els06, p. 38, Lemma 2.4.17].

The application of Lemma 4.16 to $Q^{*}=Q$ yields the following

COROllary 4.17. Let $r \geq 1$ and $d \geq 4$. Then

$$
M_{1, t} \cdots M_{d, t} \geq d^{-d}\left(\min \left\{\frac{q_{0}|t| r}{2}, \frac{1}{q|t| r}\right\}\right)^{d} .
$$

Proof. Since $|Q n|=\left|Q_{+} n\right|$ we have $|Q n| \geq q_{0}|n|$, and $|n| \geq q^{-1}|Q n|$. In the case where $M_{j, t} \leq(4 d)^{-1 / 2}$, from (4.40), $|n|_{\infty} \leq r M_{j, t}$ and $2 d^{1 / 2} \leq d$ we obtain either

$$
\begin{aligned}
& \text { (i) }|t| r d^{-1} q_{0} \leq|t| r d^{-1} \frac{|Q n|}{|n|} \leq 2 M_{j, t}, \quad \text { or } \\
& \text { (ii) } \frac{1}{|t|} \leq 2|Q n| \leq 2 q|n| \leq 2 d^{1 / 2} q|n|_{\infty} \leq q d r M_{j, t},
\end{aligned}
$$

for appropriate $(m, n) \in \mathbb{Z}^{2 d}$ depending on $j$ such that $M_{j, t}=|L(m, n, t)|_{\infty}$. Note that if $M_{j, t} \geq(4 d)^{-1 / 2}$, then $M_{j, t} \geq d^{-1}$ since $d \geq 4$. Combined with (4.42), this proves Corollary 4.17 since

$$
\min \left\{\frac{q_{0}|t| r}{2}, \frac{1}{q|t| r}\right\} \leq 1
$$

(recall that $q_{0} \leq q$ ).

In the following two lemmas we will additionally assume that the matrix $Q^{*}$ is positive definite. The smallest and largest eigenvalues of $Q^{*}$ are denoted by $q_{0}^{*}$ and $q^{*}$ respectively. 
Lemma 4.18. Let $0<\kappa<\xi<\infty$. For $g \in C^{1}[\kappa, \xi]$ such that $g \geq 0$ and $g^{\prime} \leq 0$ on $[\kappa, \xi]$, define

$$
H_{\kappa, \xi}(\tau):=H_{\kappa, \xi, Q^{*}}(\tau):=\int_{\kappa}^{\xi} I_{\left\{M_{1, t}^{*} \leq \tau\right\}} g(t) d t .
$$

Then, for all

$$
\kappa>\left(q_{0}^{*} r\right)^{-1}, \quad r^{-1} \leq \tau \leq\left(2 d^{*}\right)^{-1}
$$

with $\kappa(\tau r) \leq \xi$, where $\kappa(v)=\max \left\{\kappa,\left(2 q^{*} v d^{1 / 2}\right)^{-1}\right\}$, we have

$$
H_{\kappa, \xi}(\tau) \ll_{d^{*}} \bar{H}_{\kappa, \xi}(\tau):=\frac{q^{*}}{q_{0}^{*}} \tau^{2} \int_{\kappa(\tau r)}^{\xi} g(t) d t+\frac{1}{q_{0}^{*}} \frac{\tau}{r} g(\kappa(\tau r)) .
$$

In the case where $\kappa(\tau r)>\xi$, we have $H_{\kappa, \xi}(\tau)=0$.

Proof. See [Göt04, p. 219, Lemma 3.8].

For indicator functions $g$ Lemma 4.18 reads as follows.

Lemma 4.19. Let $\lambda$ denote the Lebesgue measure. There exists a constant $c\left(d^{*}\right)$ depending on $d^{*}$ only such that for any $r \geq 1, \tau>0$ and $\xi>\kappa$,

$$
I(\tau):=\lambda\left\{t \in[\kappa, \xi]: M_{1, t}^{*} \leq \tau\right\} \leq c\left(d^{*}\right)\left(\frac{q^{*}}{q_{0}^{*}} \tau^{2}(\xi-\kappa)+\frac{1}{q_{0}^{*}} \tau r^{-1}\right) .
$$

Proof. See [Göt04, p. 222, Lemma 3.9].

We now return to a general (not necessarily positive definite) non-degenerate, symmetric, real $d \times d$ matrix $Q$, to the corresponding norm $|L|_{\infty}$ (see (4.32)) and the associated successive minima $M_{j, t}$ (see (4.31)).

In the following we assume that $Q$ is a block-type matrix, that is, there exist positive definite matrices $Q^{+} \in \mathrm{GL}\left(\mathbb{R}^{d^{+}}\right)$and $Q^{-} \in \mathrm{GL}\left(\mathbb{R}^{d^{-}}\right)$with $d^{+}+d^{-} \geq 5$ such that

$$
Q=\left(\begin{array}{cc}
Q^{+} & 0 \\
0 & -Q^{-}
\end{array}\right)
$$

We denote the corresponding successive minima of the norm $F^{ \pm}(\cdot)$, defined by the analogue of (4.27) and (4.30) for $Q^{ \pm}$, for a fixed $t$, by $M_{j, t}^{ \pm}$, $j=1, \ldots, d^{ \pm}$. Thus, we can write

$$
M_{j, t}^{ \pm}=\left|L^{ \pm}(m, n, t)\right|_{\infty}
$$

for some $m, n \in \mathbb{Z}^{d^{ \pm}}$, where

$L^{ \pm}(m, n, t)=\left(r\left(m_{1}-t\left(Q^{ \pm} n\right)_{1}\right), \ldots, r\left(m_{d^{ \pm}}-t\left(Q^{ \pm} n\right)_{d^{ \pm}}\right), \frac{1}{r} n_{1}, \ldots, \frac{1}{r} n_{d^{ \pm}}\right)$.

As in (4.33) we have

$$
M_{j, t}^{ \pm}=M_{j,-t}^{ \pm}, \quad j=1, \ldots, d^{ \pm}, t \in \mathbb{R} .
$$


In this special case there is a simple relation between the first successive minimum of $Q$ and those of $Q^{+}$and $Q^{-}$.

Lemma 4.20. For $t \in \mathbb{R}$,

$$
M_{1, t} \geq \min \left\{M_{1, t}^{+}, M_{1, t}^{-}\right\}
$$

In particular, for $\tau \in \mathbb{R}$,

$$
I_{\left\{M_{1, t} \leq \tau\right\}} \leq I_{\left\{M_{1, t}^{+} \leq \tau\right\}}+I_{\left\{M_{1, t}^{-} \leq \tau\right\}} .
$$

Proof. Choose $(m, n)=\left(\left(\begin{array}{c}m_{+} \\ m_{-}\end{array}\right),\left(\begin{array}{l}n_{+} \\ n_{-}\end{array}\right)\right) \in \mathbb{Z}^{d} \backslash 0$ such that $M_{1, t}=$ $|L(m, n, t)|_{\infty}$. It is easy to see that

$$
M_{1, t}=|L(m, n, t)|_{\infty}=\max \left\{\left|L^{+}\left(m_{+}, n_{+}, t\right)\right|_{\infty},\left|L^{-}\left(m_{-}, n_{-},-t\right)\right|_{\infty}\right\} .
$$

Since $(m, n) \neq 0$, it follows that $\left(m_{+}, n_{+}\right) \neq 0$ or $\left(m_{-}, n_{-}\right) \neq 0$ and hence by $(4.47)$,

$$
\left|L^{+}\left(m_{+}, n_{+}, t\right)\right|_{\infty} \geq M_{1, t}^{+} \quad \text { or } \quad\left|L^{-}\left(m_{-}, n_{-},-t\right)\right|_{\infty} \geq M_{1,-t}^{-}=M_{1, t}^{-} .
$$

This proves (4.48).

Corollary 4.21. Again, let $\lambda$ denote the Lebesgue measure. Then there exists a constant $c=c(d)>1$ depending on $d$ only such that for any $r \geq 1$, $\tau>0$ and $\xi>\kappa$,

$$
I(\tau):=\lambda\left\{t \in[\kappa, \xi]: M_{1, t} \leq \tau\right\} \leq c\left(\frac{q}{q_{0}} \tau^{2}(\xi-\kappa)+\frac{1}{q_{0}} \tau r^{-1}\right) .
$$

Proof. Using Lemmas 4.19 and 4.20 we obtain

$$
\begin{aligned}
I(\tau) & \leq \int\left(I_{\left\{M_{1, t}^{+} \leq \tau\right\}}+I_{\left\{M_{1, t}^{-} \leq \tau\right\}}\right) \lambda(d t) \\
& \leq\left(c\left(d^{+}\right)+c\left(d^{-}\right)\right)\left(\frac{q}{q_{0}} \tau^{2}(\xi-\kappa)+\frac{1}{q_{0}} \tau r^{-1}\right),
\end{aligned}
$$

where we have used the fact that $q$ (resp. $q_{0}$ ) is greater (resp. smaller) than the largest (resp. smallest) eigenvalue of $Q^{+}$and $Q^{-}$. Taking $c:=$ $\max _{d^{+}, d^{-} \in \mathbb{N}, d^{+}+d^{-}=d}\left(c\left(d^{+}\right)+c\left(d^{-}\right)\right)$completes the proof. Set

Lemma 4.22. Let $M(t)=M_{1, t} \cdots M_{d, t}, \gamma=\gamma(\kappa, \xi)=r^{d} \inf _{\kappa \leq t \leq \xi} M(t)$.

$$
D=\max \left\{(2 d)^{-d} r^{d}, \gamma\right\} \quad \text { and } \quad G(\kappa, \xi)=\int_{\kappa}^{\xi} g(t) d t
$$

for $0<\kappa<\xi \leq \infty$ and let $g(t)$ and $\kappa(v)$ be as in Lemma 4.18. For $\kappa>\xi$ define $G(\kappa, \xi)=0$. Then 


$$
\begin{aligned}
I_{\kappa, \xi}:= & \int_{\kappa}^{\xi} \frac{g(t)}{M(t)^{1 / 2}} d t \\
\ll_{d} & q_{0}^{-1} r^{d / 2-2} \int_{\gamma}^{D} v^{-1 / 2+1 / d}\left(q v^{1 / d} G\left(\kappa\left(v^{1 / d}\right), \xi\right)+g\left(\kappa\left(v^{1 / d}\right)\right)\right) \frac{d v}{v} \\
& \quad+G(\kappa, \xi) .
\end{aligned}
$$

Proof. We generalize the proof in [Göt04, p. 222, Lemma 3.10]. Write $\bar{\gamma}:=\inf _{\kappa \leq t \leq \xi} M(t)$ and $c_{d}=(2 d)^{-d}$. If $\bar{\gamma} \geq c_{d}$, then $I_{\kappa, \xi} \ll_{d} G(\kappa, \xi)$ and (4.49) is obvious. In the case

$$
\bar{\gamma}<c_{d}
$$

we define

$$
J_{\kappa, \xi}(v):=\int_{\kappa}^{\xi} g(t) I_{\{M(t) \leq v\}} d t
$$

for $0<\kappa<\xi$. Since $M_{j, t} \leq M_{d, t} \ll_{d} 1$, for $j=1, \ldots, d$, by Lemma 4.14, there exists a constant $\bar{M}$ depending on $d$ only such that $M(t) \leq \bar{M}$ for all $t$. Therefore we have, for all $t \in[\kappa, \xi]$,

$$
M(t)^{-1 / 2}=\int_{\bar{\gamma}}^{\bar{M}} \varepsilon^{-1 / 2} d I_{\{M(t) \leq \varepsilon\}} .
$$

Hence, Fubini's theorem implies

$$
I_{\kappa, \xi}=\int_{\bar{\gamma}}^{\bar{M}} \varepsilon^{-1 / 2} d J_{\kappa, \xi}(\varepsilon) .
$$

Splitting the integral $I_{\kappa, \xi}$ into the part where $\varepsilon \leq c_{d}$ and its complement, we obtain

$$
I_{\kappa, \xi} \leq \int_{\bar{\gamma}}^{c_{d}} \varepsilon^{-1 / 2} d J_{\kappa, \xi}(\varepsilon)+c_{d}^{-1 / 2} \int_{\kappa}^{\xi} g(t) d t .
$$

Using partial integration we have, by (4.50) and the definition of $\bar{\gamma}$,

$$
\begin{aligned}
I_{\kappa, \xi} \leq & c_{d}^{-1 / 2} \underbrace{J_{\kappa, \xi}\left(c_{d}\right)}_{=G(\kappa, \xi)}-\bar{\gamma}^{-1 / 2} \underbrace{J_{\kappa, \xi}(\bar{\gamma})}_{=0}+\frac{1}{2} \int_{\bar{\gamma}}^{c_{d}} \varepsilon^{-3 / 2} J_{a, b}(\varepsilon) d \varepsilon \\
& +c_{d}^{-1 / 2} G(\kappa, \xi) \\
= & \frac{1}{2} \int_{\bar{\gamma}}^{c_{d}} \varepsilon^{-3 / 2} J_{a, b}(\varepsilon) d \varepsilon+2 c_{d}^{-1 / 2} G(\kappa, \xi) .
\end{aligned}
$$


Furthermore, $M(t) \geq\left(M_{1, t}\right)^{d} \geq r^{-d}$ (see (4.29)) implies, together with Lemma 4.20,

$$
\begin{aligned}
J_{\kappa, \xi}(\varepsilon) & \leq \int_{\kappa}^{\xi} g(t) I_{\left\{\left(M_{1, t}\right)^{d} \leq \varepsilon\right\}} d t=\int_{\kappa}^{\xi} g(t) I_{\left\{M_{1, t} \leq \varepsilon^{1 / d}\right\}} d t \\
& \leq \int_{\kappa}^{\xi} g(t) I_{\left\{M_{1, t}^{+} \leq \varepsilon^{1 / d}\right\}} d t+\int_{\kappa}^{\xi} g(t) I_{\left\{M_{1, t}^{-} \leq \varepsilon^{1 / d}\right\}} d t \\
& =H_{\kappa, \xi, Q^{+}}\left(\varepsilon^{1 / d}\right)+H_{\kappa, \xi, Q^{-}}\left(\varepsilon^{1 / d}\right),
\end{aligned}
$$

where $H_{\kappa, \xi, Q^{ \pm}}$is defined as in (4.43) in Lemma 4.18. The smallest and largest eigenvalues of $Q^{ \pm}$are denoted by $q_{0}^{ \pm}$and $q^{ \pm}$, respectively.

Since $r^{-d} \leq \varepsilon \leq c_{d}$ and hence $r^{-1} \leq \varepsilon^{1 / d} \leq(2 d)^{-1} \leq\left(2 d^{ \pm}\right)^{-1}$, Lemma 4.18 can be applied and by changing the variable $v=r^{d} \varepsilon$ we obtain

$$
\begin{aligned}
& \int_{\bar{\gamma}}^{c_{d}} \varepsilon^{-3 / 2} H_{\kappa, \xi, Q^{ \pm}}\left(\varepsilon^{1 / d}\right) d \varepsilon \\
& \quad \leq c\left(d^{ \pm}\right) \int_{\bar{\gamma}}^{c_{d}} \varepsilon^{-3 / 2}\left(\frac{q^{ \pm}}{q_{0}^{ \pm}} \varepsilon^{2 / d} G\left(\kappa\left(\varepsilon^{1 / d} r\right), \xi\right)+\frac{1}{q_{0}^{ \pm}} \frac{\varepsilon^{1 / d}}{r} g\left(\kappa\left(\varepsilon^{1 / d} r\right)\right)\right) d \varepsilon \\
& \quad \leq c\left(d^{ \pm}\right) \int_{\gamma}^{D} r^{3 d / 2-2} v^{-3 / 2+1 / d}\left(\frac{q^{ \pm}}{q_{0}^{ \pm}} v^{1 / d} G\left(\kappa\left(v^{1 / d}\right), \xi\right)+\frac{1}{q_{0}^{ \pm}} g\left(\kappa\left(v^{1 / d}\right)\right)\right) r^{-d} d v \\
& \quad=r^{d / 2-2} c\left(d^{ \pm}\right) \int_{\gamma}^{D} v^{-1 / 2+1 / d}\left(\frac{q^{ \pm}}{q_{0}^{ \pm}} v^{1 / d} G\left(\kappa\left(v^{1 / d}\right), \xi\right)+\frac{1}{q_{0}^{ \pm}} g\left(\kappa\left(v^{1 / d}\right)\right)\right) \frac{d v}{v} .
\end{aligned}
$$

Analyzing the proof of Lemma 4.18 we may assume that the constant $c(d)$ is increasing in $d$. Since $q_{0}=\min \left\{q_{0}^{+}, q_{0}^{-}\right\}$and $q=\max \left\{q^{+}, q^{-}\right\}$, we have

$$
\begin{aligned}
& \int_{\bar{\gamma}}^{c_{d}} \varepsilon^{-3 / 2} H_{\kappa, \xi, Q^{ \pm}}\left(\varepsilon^{1 / d}\right) d \varepsilon \\
& \leq r^{d / 2-2} c(d) \int_{\gamma}^{D} v^{-1 / 2+1 / d}\left(\frac{q}{q_{0}} v^{1 / d} G\left(\kappa\left(v^{1 / d}\right), \xi\right)+\frac{1}{q_{0}} g\left(\kappa\left(v^{1 / d}\right)\right)\right) \frac{d v}{v} .
\end{aligned}
$$

Thus we conclude, by using (4.52)-(4.54), that

$$
\begin{aligned}
& I_{\kappa, \xi} \ll_{d} r^{d / 2-2} \int_{\gamma}^{D} v^{-1 / 2+1 / d}\left(\frac{q}{q_{0}} v^{1 / d} G\left(\kappa\left(v^{1 / d}\right), \xi\right)+\frac{1}{q_{0}} g\left(\kappa\left(v^{1 / d}\right)\right)\right) \frac{d v}{v} \\
& +G(\kappa, \xi),
\end{aligned}
$$

which proves (4.49). This completes the proof of Lemma 4.22. 
Lemma 4.23. Let $0<\kappa<\xi<\infty$ and assume that $Q$ is irrational. Then

$$
\lim _{r \rightarrow \infty} \inf _{t \in[\kappa, \xi]}\left(r M_{1, t}\right) \cdots\left(r M_{d, t}\right)=\infty .
$$

Proof. See [Göt04, p. 224, Lemma 3.11] or [Els06, p. 47, Lemma 2.4.24].

Acknowledgements. I would like to thank Prof. Dr. Friedrich Götze for drawing my attention to this topic, for various fruitful discussions and many valuable suggestions. Furthermore, I am grateful to the DFG-CRC 701 for financial support. This paper is a part of my PhD thesis [Els06].

\section{References}

[BG97] V. Bentkus and F. Götze, On the lattice point problem for ellipsoids, Acta Arith. 80 (1997), 101-125.

[BG99] - , - Lattice point problems and distribution of values of quadratic forms, Ann. of Math. (2) 150 (1999), 977-1027.

[Cas78] J. W. S. Cassels, Rational Quadratic Forms, London Math. Soc. Monogr. 13, Academic Press, London, 1978.

[DM93] S. G. Dani and G. A. Margulis, Limit distributions of orbits of unipotent flows and values of quadratic forms, in: I. M. Gel'fand Seminar, Adv. Soviet Math. 16, Amer. Math. Soc., Providence, RI, 1993, 91-137.

[Dav58] H. Davenport, Indefinite quadratic forms in many variables. II, Proc. London Math. Soc. (3) 8 (1958), 109-126.

[DH46] H. Davenport and H. Heilbronn, On indefinite quadratic forms in five variables, J. London Math. Soc. 21 (1946), 185-193.

[DL72] H. Davenport and D. J. Lewis, Gaps between values of positive definite quadratic forms, Acta Arith. 22 (1972), 87-105.

[Els06] G. Elsner, Distributions of values of indefinite forms and higher-order spectral estimates for finite Markov chains, $\mathrm{PhD}$ thesis, 2006.

[EMM98] A. Eskin, G. Margulis, and S. Mozes, Upper bounds and asymptotics in a quantitative version of the Oppenheim conjecture, Ann. of Math. (2) 147 (1998), $93-141$.

[Göt04] F. Götze, Lattice point problems and values of quadratic forms, Invent. Math. 157 (2004), 195-226.

[Jar28] V. Jarník, Über Gitterpunkte in mehrdimensionalen Ellipsoiden, Math. Ann. 100 (1928), 699-721.

[Mar89] G. A. Margulis, Discrete subgroups and ergodic theory, in: Number Theory, Trace Formulas and Discrete Groups (Oslo, 1987), Academic Press, Boston, MA, 1989, 377-398.

[Mar97] -, Oppenheim conjecture, in: Fields Medallists' lectures, World Sci. Ser. 20th Century Math., World Sci., River Edge, NJ, 1997, 272-327.

[Mum83] D. Mumford, Tata Lectures on Theta. I, Progr. Math. 28, Birkhäuser Boston, Boston, MA, 1983.

[Opp29] A. Oppenheim, The minima of indefinite quaternary quadratic forms, Proc. Nat. Acad. Sci. USA 15 (1929), 724-727. 
[Opp31] A. Oppenheim, The minima of indefinite quaternary quadratic forms, Ann. of Math. (2) 32 (1931), 271-298.

Faculty of Mathematics

University of Bielefeld

P.O. Box 100131

33501 Bielefeld, Germany

E-mail: GUIDE@math.uni-bielefeld.de 